Ricardo H. Nochetto - Tobias von Petersdorff .

Chen-Song Zhang

\title{
A Posteriori Error Analysis for A Class of Integral Equations and Variational Inequalities
}

June 23, 2009

\begin{abstract}
We consider elliptic and parabolic variational equations and inequalities governed by integro-differential operators of order $2 s \in(0,2]$. Our main motivation is the pricing of European or American options under Lévy processes, in particular pure jump processes or jump diffusion processes with tempered stable processes. The problem is discretized using piecewise linear finite elements in space and the implicit Euler method in time. We construct a residual-type a posteriori error estimator which gives a computable upper bound for the actual error in $H^{s}$-norm. The estimator is localized in the sense that the residuals are restricted to the discrete non-contact region. Numerical experiments illustrate the accuracy of the space and time estimators, and show that they can be used to measure local errors and drive adaptive algorithms.
\end{abstract}

\section{Introduction}

Integral equations and variational inequalities with integral operators are important in various applications. We now mention a few of them, the first one being our main motivation. It is worth observing that our results are valid for all of these applications.

- Option pricing with jump processes: a jump process is modeled by an integral operator and leads to a parabolic equation (European option) or a parabolic variational inequality (American option) [65,24]; see section 2 for details.

- Boundary integral equations and inequalities: they are obtained from elliptic partial differential equations (PDEs) in a bounded or exterior domain $[25,36,51]$, perhaps with unilateral constraint on the boundary (Signorini problem) [56].

\footnotetext{
Ricardo H. Nochetto

Department of Mathematics and Institute of Physical Science and Technology, University of Maryland, College Park, MD 20742 USA. This author has been partially supported by NSF Grants DMS-0505454 and DMS-0807811.

Tobias von Petersdorff

Department of Mathematics, University of Maryland, College Park, MD 20742 USA.

Chen-Song Zhang

Department of Mathematics, Penn State University, State College, PA 16802 USA. This author has been partially supported by NSF Grant DMS-0505454.
} 
- Fractional Laplacian: boundary control problems [28,14], surface frame propagation and quasigeostrophic flow models $[15,23]$ are all problems governed by the fractional Laplacian.

We consider a linear integral operator $\mathcal{I}$ of order $\rho \in(0,2)$ on a bounded domain $\Omega \subset \mathbb{R}^{d}$. For $s \in(0,1]$ we define the Sobolev space $\widetilde{H}^{s}(\Omega)=\left\{\left.u\right|_{\Omega}: u \in H^{s}\left(\mathbb{R}^{d}\right),\left.u\right|_{\mathbb{R}^{d} \backslash \Omega}=0\right\}$; see, e.g., $[50,44]$. In particular we consider integral operators of the form

$$
\mathcal{I} u=K * u \quad \text { with } \quad K(x)=\frac{g(x)}{\|x\|^{d+\rho}},
$$

where the convolution integral is defined by a suitable regularization and $\rho=2 s$; see sections 2 and 3.2. A special case of this is the fractional Laplacian $(-\Delta)^{s}$.

The operator $\mathcal{A}$ is either an integral operator $\mathcal{A}=\mathcal{I}$ of order $2 s \in(0,2)$, or an integrodifferential operator $\mathcal{A}=\mathcal{D}+\mathcal{I}$ of order $2 s=2$ where $\mathcal{D}$ is a second-order differential operator; hence, $2 s$ will always indicate the order of $\mathcal{A}$. In both cases we define the energy space $\mathcal{V}=\widetilde{H}^{s}(\Omega)$ and assume that $\mathcal{A}: \mathcal{V} \rightarrow \mathcal{V}^{*}$ is continuous and coercive; see section 3.1.

We examine the parabolic equation

$$
u_{t}(t)+\mathcal{A} u(t)=f(t)
$$

with initial condition $u(0)=u_{0}$, which in weak form reads as follows: find $u(t) \in \mathcal{V}$ such that

$$
\left\langle u_{t}(t)+\mathcal{A} u(t)-f(t), v\right\rangle=0 \quad \forall v \in \mathcal{V} .
$$

If we add the obstacle constraint $u \geq \chi$, then (1.2) becomes the parabolic variational inequality

$$
\left\langle u_{t}(t)+\mathcal{A} u(t)-f(t), u(t)-v\right\rangle \leq 0 \quad \forall v \in \mathcal{V} \text { with } v \geq \chi
$$

see section 3 for precise definitions and assumptions. Note that, for each time $t$, there is a contact region $\{u=\chi\}$, and a non-contact region $\{u>\chi\}$ where $u_{t}(t)+\mathcal{A} u(t)=f(t)$, separated by a free boundary. We also discuss the corresponding elliptic problems.

For the space discretization we use piecewise linear finite elements in space over a mesh which does not depend on time, but may be graded. For the time discretization we use the implicit Euler method where the time partition may also be nonuniform. One main objective is to construct a computable a posteriori error estimator: the estimator uses the computed discrete solution, operator and data, and gives an upper bound of the error, measured in the norm of $\mathcal{V}$ in the time-independent case, and measured in $L^{2}(0, T ; \mathcal{V})$ in the time-dependent case. We are thus after error control of (1.2) and (1.3) rather than their efficient and fast solution.

For elliptic PDEs, a posteriori error estimators are an essential part of practical and reliable numerical methods: they indicate whether a computed solution is acceptable, or whether further mesh refinement and coarsening may enhance it. This is achieved by splitting the error estimator into local error indicators, which are in turn employed as a measure of local resolution. Error estimators are particularly important if the solution exhibits singularities. For elliptic PDEs the theory of error estimators and adaptive algorithms is now well established for coercive operators $[61,1]$. In particular, it has been shown that adaptive algorithms achieve optimal convergence rates for a large class of singular solutions [46]. On the other hand, the theory for parabolic PDEs is much less developed $[21,40,54]$.

In extending the a posteriori error analysis from elliptic equations to more general problems, including integral operators, variational inequalities, and parabolic problems, we face specific challenges. We describe them now in turn. 
- Integral operators: In this case the operator $\mathcal{A}$ is nonlocal, and the energy norm is hard to compute as the norms of fractional Sobolev spaces $\widetilde{H}^{s}(\Omega)$ cannot be simply broken up into local parts over subdomains. This issue has been studied by many others [63,64,20,18,19,29-31]. In particular, Faermann [29-31] show how to localize fractional Sobolev norms. We provide an alternative approach based on stars (element patches) which yields a natural localization. An additional issue is that the residuals are singular at the boundaries between elements, so that the $L^{2}$-norm does not always exist. Our estimator uses $L^{p}$-norms of the residual, and for $\rho>\frac{3}{2}$ values of $p<2$ are required [5]. This seems to be a new result even for linear elliptic equations. We also discuss a method for the efficient numerical evaluation of the resulting singular integrals.

- Elliptic variational inequalities: The location of the free boundary is unknown, and the standard residual estimator is only appropriate in the non-contact region. Fierro and Veeser [33] constructed an estimator for differential operators of order 2 which is properly localized: the estimator only uses the residual in a discrete non-contact region, and resolves the obstacle only in the discrete contact set. This is accomplished upon dealing with estimators on stars (rather than elements) and a suitable definition of discrete contact set. We follow a similar approach for integral operators in sections 5 and 6 . Note that no special assumptions are needed about the geometry of the exact free boundary, which, in the multidimensional case, can indeed be very complicated. Even in the simplest case of a standard call or put option in one dimension, where the free boundary consists only of a single point, one cannot simply use the error estimator from the elliptic case as the exact location is unknown.

- Parabolic problems: Additional singularities in space and time occur at $t=0$, depending on the initial data, and at the domain boundary. In [45] an error estimator was constructed which measures the space discretization error via the elliptic estimator of Fierro and Veeser [33] and the time discretization error via the estimator of Nochetto, Savaré, and Verdi [47] for the implicit Euler method. We extend this approach to integral equations in section 7 .

- Singularities at the free boundary: For both elliptic and parabolic variational inequalities with integral operators, the solution can exhibit singularities [42] at the free boundary which are strong enough to reduce the convergence rate for piecewise linear elements; this does not happen in the case of a differential operator $[33,49,45]$. This makes error estimators and adaptivity particularly important as we require mesh refinement and coarsening at a priori unknown locations (in contrast to linear PDEs where singularities can only occur at points where the domain boundary or right hand side function are nonsmooth). We explore these issues computationally in section 8 .

We organize the paper as follows. In $\S 2$ we briefly discuss the option pricing problem which is the main motivation for our results. In $\S 3$ we describe the class of operators under consideration and a suitable functional framework for their study. In $\S 4$ we consider linear elliptic integral equations and introduce our technique for estimating the error in terms of $L^{p}$-norms of the residual. In $\S 5$ we introduce the elliptic variational inequalities for integro-differential operators of order $2 s$, along with Lagrange multipliers, both continuous and discrete, and the Galerkin functional. In $\S 6$ we derive upper a posteriori error estimates for the elliptic case; this extends the results of Fierro and Veeser [33] to fractional Sobolev norms $H^{s}$ with $s<1$. In $\S 7$ we tackle the parabolic case and thereby extend the a posteriori error estimates in [45] to $s<1$. We conclude in $\S 8$ with several numerical experiments for both elliptic and parabolic equations and variational inequalities for $d=1$ and $s \leq 1$. We discuss how to compute the residuals and their $L^{p}$-norms. 


\section{Financial Problem}

We now give a very brief description of the option pricing problem in the case of one asset $(d=1)$. For more details see e.g. [24]. Numerical methods for pricing American options have been discussed by many researchers (see $[17,27,2-4,42,41,22]$ and references therein).

We consider an asset (e.g., stock) with known initial price $S(0)$ and let $r \geq 0$ denote the interest rate. The price $S(t)$ for $t \in[0, T]$ is modeled by a stochastic process under the so-called risk-neutral measure, i.e., $e^{-r t} S(t)$ is a martingale. We define the $\log$-price $X(t)=\log S(t)$ and assume that $X(t)-X(0)$ is a Lévy process. A European option is a contract which pays the amount $G(X(T))$ at maturity time $T$ where $G: \mathbb{R} \rightarrow \mathbb{R}$ is a given payoff function. An American option is a contract which pays the amount $G(X(t))$ at any time $t \in[0, T]$ the holder of the option chooses the so-called exercise time.

Given the known initial log-price $X(0)$ we want to find the fair market price of the option. Let $u(x, T-t)$ denote the option price at time $t$ if the current asset log-price is $x=X(t)$. Hence $u(x, 0)=G(x)$ and $u(X(0), T)$ is the desired option price. For a European option the function $u(x, t)$ satisfies the Kolmogorov equation (1.2) with

$$
\mathcal{A} u=-\mathcal{B} u+r\left(u-u_{x}\right)
$$

and initial condition $u(x, 0)=G(x)$. Here $\mathcal{B}$ is the infinitesimal generator of the stochastic process $X(t)$. If there are no jumps, the process $X(t)$ must be a Brownian motion with drift and we have

$$
\mathcal{B} u=\sigma^{2}\left(u_{x x}-u_{x}\right)
$$

A pure jump process is characterized by the so-called jump density $K(z)$. The expected number of jumps in a set $B \subset \mathbb{R}$ per unit time is then given by $\int_{B} K(z) d z$. The function $\min \left\{1,|z|^{2}\right\} K(z)$ must be integrable and $\mathcal{B}$ is given by

$$
\mathcal{B} u=\int_{\mathbb{R}^{d}} K(z)\left[u(x+z)-u(x)-\left(e^{z}-1\right) u_{x}(x)\right] d z
$$

(assuming sufficiently fast decay of $K(z)$ for $|z| \rightarrow \infty$ ). This is actually a regularized form of the convolution (1.1) with the kernel function $K(z)$. A relevant example is the so-called tempered stable process, also known as CGMY process, where

$$
K(z)=\frac{g(z)}{|z|^{1+\rho}}, \quad g(z)= \begin{cases}e^{G_{+}|z|} & \text { for } z \geq 0 \\ e^{G_{-}|z|} & \text { for } z<0\end{cases}
$$

and $\rho \in(0,2)$ is the order of the integral operator $\mathcal{B}$; see $[11,16]$. For a general Lévy process $\mathcal{B} u$ is the sum of the operators in (2.2) and (2.3).

For an American option, the function $u(x, t)$ satisfies the variational parabolic inequality (1.3) with $\chi(x)=G(x)$ instead of (1.2). The contact region specifies the optimal exercise strategy: if at time $t$ the current log-price $x=X(t)$ of the stock is in the contact region one should exercise the option, otherwise one should wait. The free boundary, i.e. the boundary of the contact region, is thus a crucial unknown.

For multiple assets, we have a process $x=X(t)$ with values in $\mathbb{R}^{d}$, and a function $u(x, t)$ satisfying an elliptic PDE in case of Brownian motion, or an integral equation in case of a pure jump process. For several dimensions we will only consider jump densities of the form

$$
K(x)=\frac{g(x)}{|x|^{d+\rho}}
$$


where $g(x)$ is smooth and exponentially decaying for $|x| \rightarrow \infty$. Note that there are so-called Lévy copulas which lead to jump densities with anisotropic singular behavior as described in [32]; this case is not covered in this paper.

The original problem uses $x \in \mathbb{R}^{d}$, but because of the exponential decay of the kernel function $K(z)$ one can truncate the problem to a bounded domain $\Omega$ and consider functions $u \in \mathcal{V}=$ $\widetilde{H}^{s}(\Omega)$. This leads to an error which decays exponentially with the size of the truncated domain; see e.g. [43]. The problem on a bounded domain $\Omega$ corresponds to a so-called barrier option which becomes worthless as soon as the $\log$-price $X(t)$ leaves $\Omega$.

\section{Operators and Variational Setting}

In the rest of the paper, we write $A \lesssim B$ instead of $A \leq C B$ with a generic geometric constant $C$ independent of meshsize, time-step, and various functions involved, as well as $A \approx B$ in place of $A \lesssim B \lesssim A$. Let $\mathcal{H}, \mathcal{V}$ be Hilbert spaces so that $\mathcal{V}$ is dense in $\mathcal{H}$ with a continuous embedding $\mathcal{V} \hookrightarrow \mathcal{H}$. We identify $\mathcal{H}$ with its dual $\mathcal{H}^{*}$ and obtain the usual triple $\mathcal{V} \hookrightarrow \mathcal{H} \sim \mathcal{H}^{*} \hookrightarrow \mathcal{V}^{*}$. We first describe both the time-independent and time-dependent problems in an abstract setting with linear operator $\mathcal{A}: \mathcal{V} \rightarrow \mathcal{V}^{*}$. We then specify the class of integro-differential operators $\mathcal{A}$ under consideration.

\subsection{Abstract Variational Setting}

Let $\mathcal{A}: \mathcal{V} \rightarrow \mathcal{V}^{*}$ be a linear operator which is continuous and coercive, i.e., there is $C_{\mathcal{A}}, \kappa_{\mathcal{A}}>0$ such that for all $v, w \in \mathcal{V}$

$$
\begin{array}{rlrl}
\langle\mathcal{A} v, w\rangle & \leq C_{\mathcal{A}}\|v\|_{\mathcal{V}}\|w\|_{\mathcal{V}} & & \text { (continuity) } \\
\langle\mathcal{A} v, v\rangle & \geq \kappa_{\mathcal{A}}\|v\|_{\mathcal{V}}^{2} & \text { (coercivity) }
\end{array}
$$

Hereafter, $\langle\cdot, \cdot\rangle$ stands for the duality pairing between $\mathcal{V}^{*}$ and $\mathcal{V}$. We define the bilinear form $a(v, w):=\langle\mathcal{A} v, w\rangle$ and the energy norm $\|v\|:=a(v, v)^{\frac{1}{2}}=\langle\mathcal{A} v, v\rangle^{\frac{1}{2}}$. The induced norm on the dual space $\mathcal{V}^{*}$ will be denoted by $\|\cdot\|_{*}$. Note that (3.1a), (3.1b) imply that $\mathcal{A}$ satisfies a strong sector condition

$$
|\langle\mathcal{A} u, v\rangle| \leq 2 \gamma\|u\|\|v\| .
$$

We clearly have $\frac{1}{2} \leq \gamma \leq \frac{C_{\mathcal{A}}}{2 \kappa_{\mathcal{A}}}$, but this may be pessimistic since $\gamma=\frac{1}{2}$ in the symmetric case.

Let $f \in \mathcal{V}^{*}$. We consider the following time independent problem (elliptic variational inequality): find $u \in \mathcal{K}$ such that

$$
\langle\mathcal{A} u-f, u-v\rangle \leq 0 \quad \forall v \in \mathcal{K},
$$

where $\mathcal{K}$ is a closed convex subset of $\mathcal{V}$. It is well known that this problem has a unique solution; see, e.g., [37].

Similarly, for $f \in L^{2}\left(0, T ; \mathcal{V}^{*}\right)$ and $u_{0} \in \mathcal{K}(0)$, we consider the following time dependent problem (parabolic variational inequality): Find $u \in C([0, T] ; \mathcal{H}) \cap L^{2}(0, T ; \mathcal{V})$ with the initial condition $u(0)=u_{0}$ such that for almost every $t \in[0, T]$ there holds $u(t) \in \mathcal{K}(t)$ and

$$
\left\langle u_{t}(t)+\mathcal{A} u(t)-f(t), u(t)-v\right\rangle \leq 0 \quad \forall v \in \mathcal{K}(t) .
$$

Note that the initial condition and the time derivative have to be defined in a weak sense; see [7] where actually a setting with more general $u_{0}$ and $f(t)$ is considered. On the other hand, if we have $f \in L^{2}(0, T ; \mathcal{H})$ there holds $u \in H^{1}(0, T ; \mathcal{H})$ so that the initial condition and (3.4) hold in the classical sense; see [13]. 


\subsection{Spaces and Operators}

We now specify the spaces $\mathcal{H}, \mathcal{V}$, convex set $\mathcal{K}$ and classes of operators $\mathcal{A}$ under consideration.

Let $\Omega \subset \mathbb{R}^{d}$ be a bounded polyhedral domain with boundary $\Gamma$ and let $s \in(0,1]$. We first define the space of functions on $\Omega$ so that their extensions by zero are in $H^{s}(\Omega)$ :

$$
\widetilde{H}^{s}(\Omega)=\left\{\left.u\right|_{\Omega}: u \in H^{s}\left(\mathbb{R}^{d}\right),\left.u\right|_{\mathbb{R}^{d} \backslash \Omega}=0\right\} .
$$

Another space is obtained by restricting $H^{s}\left(\mathbb{R}^{d}\right)$ functions to $\Omega$ :

$$
H^{s}(\Omega):=\left\{\left.u\right|_{\Omega}: u \in H^{s}\left(\mathbb{R}^{d}\right)\right\} .
$$

Note that $\widetilde{H}^{s}(\Omega):=\left[L^{2}(\Omega), H_{0}^{1}(\Omega)\right]_{s}$ and $H^{s}(\Omega):=\left[L^{2}(\Omega), H^{1}(\Omega)\right]_{s}$ for $s \in(0,1]$ for the interpolation spaces with the induced norm; see $[9,44]$ for example. We define for $s>0$ the dual spaces $H^{-s}(\Omega):=\widetilde{H}^{s}(\Omega)^{*}$ and $\widetilde{H}^{-s}(\Omega):=H^{s}(\Omega)^{*}$.

Let $\omega$ be a sub-domain of $\Omega$. We define the spaces $H_{\Gamma}^{s}(\omega)$ for $s \in(0,1]$ by interpolation of

$$
H_{\Gamma}^{0}(\omega):=L^{2}(\omega) \quad \text { and } \quad H_{\Gamma}^{1}(\omega):=\left\{u \in H^{1}(\omega):\left.u\right|_{\Gamma}=0\right\} .
$$

It is then clear from their definitions that $H_{\Gamma}^{s}(\Omega)=\widetilde{H}^{s}(\Omega)$ and $H_{\Gamma}^{s}(\omega)=H^{s}(\omega)$ provided $\partial \omega \cap \Gamma$ is empty. We use $H_{\Gamma}^{s}(\omega)^{*}$ to denote the dual space of $H_{\Gamma}^{s}(\omega)$.

In American option pricing the convex set $\mathcal{K}$ corresponds to a unilateral constraint given by the payoff function $\chi \in \mathcal{V}$, namely,

$$
\mathcal{K}=\{v \in \mathcal{V}: v \geq \chi\} .
$$

To simplify the presentation, we assume that the obstacle $\chi$ is a piecewise linear function in the finite element space. More general obstacles, which may also be time-dependent, can be treated similarly; we skip the details and refer to $[45$, Section 4$]$ for details (see also $[60,49]$ ).

We now introduce the integral operators $\mathcal{I}$. Let $K \geq 0$ denote the jump density and assume it is a tempered distribution on $\mathbb{R}^{d}$ so that its Fourier transform $\hat{K}$ satisfies

$$
|\hat{K}(\xi)| \leq C(1+|\xi|)^{\rho}
$$

with $\rho<2$. Then the convolution $u \mapsto K * u$ is a continuous mapping between $H^{r}\left(\mathbb{R}^{d}\right) \rightarrow$ $H^{r-\rho}\left(\mathbb{R}^{d}\right)$ as well as $\widetilde{H}^{r}(\Omega) \rightarrow H^{r-\rho}(\Omega)$ for all $r \in \mathbb{R}$. We assume that the kernel $K(x)$ is smooth for $x \neq 0$ and satisfies

$$
\left|\partial_{x}^{\beta} K(x)\right| \leq C_{\beta}|x|^{-d-\rho-|\beta|}
$$

near $x=0$ for all multi-indices $\beta$. Examples for such processes are so-called tempered stable processes where the kernel has the form

$$
K(x)=\frac{g(x)}{|x|^{d+\rho}} \quad \text { for } x \neq 0
$$

and $g(x) \geq 0$ decays exponentially for $|x| \rightarrow \infty$; for $d=1, g(x)$ could be given by (2.4) for example. We now let $s=\rho / 2$ and define the integral operator $\mathcal{I}: \widetilde{H}^{s}(\Omega) \rightarrow H^{-s}(\Omega)$ as follows: consider a smooth function $u$ with support in $\Omega$, extended by zero to $\mathbb{R}^{d}$. If $\rho<0$, then the convolution $-K * u$ can be expressed as a classical integral

$$
\mathcal{I} u(x):=-\int_{\mathbb{R}^{d}} K(x-y) u(y) d y .
$$


However, for $\rho \geq 0$ the convolution integral does not exist in the $L^{1}$ sense, and we thus consider regularizations of the integral; see [36,39]. For $0 \leq \rho<1$ we use

$$
\mathcal{I} u(x):=-\int_{\mathbb{R}^{d}} K(x-y)(u(y)-u(x)) d y,
$$

whereas, for $1 \leq \rho<2$ we use

$$
\mathcal{I} u(x):=-\int_{\mathbb{R}^{d}} K(x-y)(u(y)-u(x)-\nabla u(x) \cdot(y-x)) d y
$$

to obtain an integrable integrand. Because of (3.6) equations (3.7)-(3.9) define a continuous operator $\mathcal{I}: \widetilde{H}^{s}(\Omega) \rightarrow H^{-s}(\Omega)$.

We now specify the class of integro-differential operators $\mathcal{A}$ by considering the following cases.

Case C1: $s=\rho / 2 \in[1 / 2,1)$. Here $\mathcal{A}$ consists of an integral operator of order $\rho$ and a differential operator of order 1 . Let $c_{1} \in \mathbb{R}^{d}, c_{0} \in \mathbb{R}$ and

$$
\mathcal{A} u:=\mathcal{I} u+c_{1} \cdot \nabla u+c_{0} u
$$

This corresponds to (2.1) with a pure jump process where $\mathcal{B}$ is given by (2.3) and (2.4). Note that for a bounded domain $\Omega$ the difference between the regularizations in (3.9) and (2.3) is just a term $c \nabla u$.

Case C2: $s=\rho / 2 \in(0,1 / 2)$. Here $\mathcal{A}$ is an integral operator of order $\rho$ :

$$
\mathcal{A} u:=\mathcal{I} u+c_{0} u
$$

For a pure jump process with kernel (2.4) the operator $\mathcal{A}$ in (2.1) contains a first order term $r u_{x}$. One can, however, eliminate this term from the parabolic problem by using the change of variable $\widetilde{x}=x-r t$, at the expense of having a time-dependent obstacle [45].

Case C3: $s=1>\rho / 2$. Here $\mathcal{A}$ consists of an integral operator of order $\rho$ and an elliptic differential operator of order 2 :

$$
\mathcal{A} u:=-\nabla \cdot(\mu \nabla u)+\mathcal{I} u+c_{1} \cdot \nabla u+c_{0} u .
$$

We assume that the matrix $\mu \in \mathbb{R}^{d \times d}$ is positive definite ( $\mu$ corresponds to the covariance matrix of the Brownian motion). This corresponds to $(2.1)$ where the process $X(t)$ is a Lévy process with nonzero diffusion part. In this case $\mathcal{B}$ is the sum of the operators in (2.2) and (2.3).

In all three cases $\mathrm{C} 1, \mathrm{C} 2, \mathrm{C} 3$ the operator $\mathcal{A}: \widetilde{H}^{s}(\Omega) \rightarrow H^{-s}(\Omega)$ is continuous and satisfies the Gårding inequality,

$$
\langle\mathcal{A} v, v\rangle \geq \kappa_{\mathcal{A}}\|v\|^{2}-c\|v\|_{L^{2}(\Omega)}^{2}
$$

with $\kappa_{\mathcal{A}}>0$ and $c \geq 0$, i.e., the operator $\mathcal{A}+c I$ is coercive ( $I$ is the identity operator). For time-independent problems we will assume that $\mathcal{A}$ is coercive, i.e., (3.13) holds with $c=0$. For time-dependent problems one can use the change of variables $\widetilde{u}(x, t):=e^{-c t} u(x, t)$ and express the problem in terms of the coercive operator $\widetilde{\mathcal{A}}=\mathcal{A}+c I$, again at the expense of having a time-dependent obstacle. This is why we assume (3.1). 


\section{Linear Integral Equation}

We first consider the simplest case of a linear integral equation $\mathcal{A} u=f$. Here $\mathcal{A}$ is the operator defined in section 3.2 and satisfies (3.1).

We focus first on cases $\mathrm{C} 1$ and $\mathrm{C} 2$, with the case $\mathrm{C} 3$ being postponed until section 4.3. In cases $\mathrm{C} 1$ and $\mathrm{C} 2$ the operator $\mathcal{A}$ is an integro-differential operator with differential part of order $\leq \rho$. Therefore, $\mathcal{A}$ is dominated by the integral operator $\mathcal{I}$ of order $\rho=2 s \in(0,2)$, and the energy space is $\widetilde{H}^{s}(\Omega)$. We derive residual-type a posteriori error estimators for the $H^{s}$-norm which are computable. The theory is based on a partition of unity and a localization result for fractional Sobolev spaces, which is also proved. We refer to Faermann [29-31] for a different approach.

We consider the integral equation: given $f \in H^{-s}(\Omega)$, find $u \in \widetilde{H}^{s}(\Omega)$ such that

$$
\mathcal{A} u=f .
$$

Because of continuity and coercivity (3.1) of $\mathcal{A}$, this problem has a unique solution satisfying

$$
\|u\|_{\widetilde{H}^{s}(\Omega)} \leq \kappa_{\mathcal{A}}^{-1}\|f\|_{H^{-s}(\Omega)} .
$$

For the discretization we define a finite-dimensional subspace $\mathbb{V}_{h}$ as follows. Let $\left\{\mathcal{T}_{h}\right\}_{h}$ be a family of shape-regular triangulations of the polyhedral domain $\Omega$. We define for $x \in \Omega$ the local meshsize $h(x)$ by $\left.h\right|_{\tau}:=h_{\tau}$ where $h_{\tau}$ denotes the diameter of an element $\tau \in \mathcal{T}_{h}$. Let $\mathcal{P}_{h}$ be the set of all nodes of the mesh $\mathcal{T}_{h}$, including the boundary nodes. Let $\mathbb{V}_{h}$ be the space of continuous piecewise linear functions $v_{h}$ over the mesh $\mathcal{T}_{h}$ with $v_{h}=0$ on the boundary of $\Omega$. Let $\left\{\psi_{z}\right\}_{z \in \mathcal{P}_{h}}$ be the canonical nodal basis of $\mathbb{V}_{h}$. For $z \in \mathcal{P}_{h}$, we define the star $\omega_{z}$ as the support of the piecewise linear nodal basis functions $\psi_{z}$, the skeleton $\gamma_{z}$ as the union of all interior sides containing $z$, and $h_{z}:=\max \left\{h(x): x \in \omega_{z}\right\}$; for $d=1, \gamma_{z}$ reduces to $\{z\}$.

We define the discrete problem as follows: find $u_{h} \in \mathbb{V}_{h}$ such that for all $v_{h} \in \mathbb{V}_{h}$

$$
\left\langle\mathcal{A} u_{h}, v_{h}\right\rangle=\left\langle f, v_{h}\right\rangle .
$$

In view of (3.1) this problem has a unique solution. The residual is the distribution

$$
r_{h}:=f-\mathcal{A} u_{h} \in H^{-s}(\Omega)
$$

which, again by (3.1), satisfies

$$
\kappa_{\mathcal{A}}\left\|u-u_{h}\right\|_{H^{s}(\Omega)} \leq\left\|r_{h}\right\|_{H^{-s}(\Omega)} \leq C_{\mathcal{A}}\left\|u-u_{h}\right\|_{H^{s}(\Omega)} .
$$

Therefore the issue at stake is how to estimate $\left\|r_{h}\right\|_{H^{-s}(\Omega)}$ in terms of computable quantities. We first want to investigate the regularity of $r_{h}$. Since the Fourier transform of the kernel satisfies (3.6) we get from [58, Theorem XI.2.5] that the pseudodifferential operator

$$
\mathcal{A}: \widetilde{W}_{p}^{t}(\Omega) \rightarrow W_{p}^{t-2 s}(\Omega)
$$

is continuous for all $t \in \mathbb{R}$ and $p \in(1, \infty)$. As $u_{h}$ is continuous piecewise linear we have that $u_{h} \in \widetilde{W}_{p}^{1+\frac{1}{p}-\varepsilon}(\Omega)$ for any $\varepsilon>0$. Hence we obtain $\mathcal{A} u_{h} \in W_{p}^{1+\frac{1}{p}-2 s-\varepsilon}(\Omega)$ and

$$
\mathcal{A} u_{h} \in L^{p}(\Omega) \quad \text { for } \frac{1}{p}>2 s-1 .
$$

Since $s \in(0,1)$ there exists $p>1$ with $\mathcal{A} u_{h} \in L^{p}(\Omega)$. 
In cases $\mathrm{C} 1$ and $\mathrm{C} 2$ the residual $r_{h}$ is just a function $R$ without any delta distributions at the interelement boundaries. It is our goal to estimate $\|R\|_{H^{-s}(\Omega)}$ in terms of local $L^{p}$-norms of the residual. This involves two steps: (1) estimate the global norm $\|R\|_{H^{-s}(\Omega)}$ in terms of local $H^{-s}$-norms; (2) estimate the local $H^{-s}$-norms by local $L^{p}$-norms. As the norm of $H^{-s}(\Omega)$ is nonlocal one cannot estimate $\|R\|_{H^{-s}(\Omega)}$ in terms of norms $\|R\|_{H^{-s}(\tau)}$ on the elements. Our technique is to use the partition of unity given by the nodal basis functions. This leads to local $H^{-s}$-norms on the stars $\omega_{z}$. Our approach leads to a different upper bound of $\|R\|_{H^{-s}(\Omega)}$ as compared with Faermann [29-31].

In case $\mathrm{C} 3$, however, the residual $r_{h}$ has delta distributions on the interelement boundaries. We will thus split $r_{h}$ into an interior residual $R$ on element interior plus jumps of the fluxes $\mu \nabla u_{h}$ on element boundaries.

\subsection{Localization of Fractional Sobolev Norms}

We first show that we can bound the global $H^{-s}(\Omega)$-norm by a sum of localized norms on $\omega_{z}$ for $s \in[0,1]$.

Lemma 1 (Localized Upper Bound of the Dual Norm) Let $G=\sum_{z \in \mathcal{P}_{h}} g_{z}$ and $g_{z} \in H_{\Gamma}^{-s}\left(\omega_{z}\right)$. For $s \in[0,1]$ there holds

$$
\|G\|_{H^{-s}(\Omega)}^{2} \leq(d+1) \sum_{z \in \mathcal{P}_{h}}\left\|g_{z}\right\|_{H_{\Gamma}^{s}\left(\omega_{z}\right)^{*}}^{2}
$$

Proof We have for $v \in \widetilde{H}^{s}(\Omega)$,

$$
|\langle G, v\rangle| \leq \sum_{z \in \mathcal{P}_{h}}\left|\left\langle g_{z}, v\right\rangle\right| \leq\left(\sum_{z \in \mathcal{P}_{h}}\left\|g_{z}\right\|_{H_{\Gamma}^{s}\left(\omega_{z}\right)^{*}}^{2}\right)^{1 / 2}\left(\sum_{z \in \mathcal{P}_{h}}\|v\|_{H_{\Gamma}^{s}\left(\omega_{z}\right)}^{2}\right)^{1 / 2} .
$$

Note that, for $s=0$ and $s=1$, we have

$$
\sum_{z \in \mathcal{P}_{h}}\|v\|_{H_{\Gamma}^{s}\left(\omega_{z}\right)}^{2} \leq(d+1)\|v\|_{\widetilde{H}^{s}(\Omega)}^{2}
$$

since at most $d+1$ of the stars $\omega_{z}$ overlap on each simplex.

For any $v \in \widetilde{H}^{s}(\Omega)$, we define the operator $\mathcal{L}: \widetilde{H}^{s}(\Omega) \rightarrow \prod_{z \in \mathcal{P}_{h}} H_{\Gamma}^{s}\left(\omega_{z}\right)$, which restricts $v$ to local patches, i.e.

$$
\mathcal{L}(v):=\left(v_{z}\right)_{z \in \mathcal{P}_{h}} \quad \text { with } \quad v_{z}(x):= \begin{cases}v(x) & x \in \omega_{z} \\ 0 & \text { otherwise. }\end{cases}
$$

For $s=0$ or $s=1,(4.4)$ gives $\|\mathcal{L}(v)\|^{2} \leq(d+1)\|v\|_{\widetilde{H}^{s}(\Omega)}^{2}$. By interpolation between $L^{2}(\Omega)$ and $H_{0}^{1}(\Omega)$, we obtain (4.4) for all $s \in[0,1]$, which in turn implies (4.3).

The following lemma provides a computable estimate of negative norms in terms of $L^{p}$-norms.

Lemma 2 (Upper Bound of Local Dual Norm) Let $g_{z} \in L^{p}\left(\omega_{z}\right)$ satisfy $\int_{\omega_{z}} g_{z}=0$ for $z \in \mathcal{P}_{h}$ such that $\partial \omega_{z} \cap \Gamma$ has measure 0 . If $s \in[0,1]$ and $1 \leq p<\infty$ satisfy $\frac{1}{p}<\frac{s}{d}+\frac{1}{2}$, then

$$
\left\|g_{z}\right\|_{H_{\Gamma}^{s}\left(\omega_{z}\right)^{*}} \lesssim h_{z}^{s+d(1 / 2-1 / p)}\left\|g_{z}\right\|_{L^{p}\left(\omega_{z}\right)}
$$


Proof If $1<q=\frac{p}{p-1} \leq \infty$ is the dual Lebesgue exponent of $p$, then we have $H_{\Gamma}^{s}\left(\omega_{z}\right) \hookrightarrow L^{q}\left(\omega_{z}\right)$ because $s-\frac{d}{2}>-\frac{d}{q}$, whence by duality $L^{p}\left(\omega_{z}\right) \hookrightarrow H_{\Gamma}^{s}\left(\omega_{z}\right)^{*}$. We split the proof in two steps according to whether $\partial \omega_{z} \cap \Gamma$ has measure 0 or not.

$1 \partial \omega_{z} \cap \Gamma$ has measure 0 . Since $\int_{\omega_{z}} g_{z}=0$, for any $v \in H_{\Gamma}^{s}\left(\omega_{z}\right)$ and $C_{z} \in \mathbb{R}$, we have

$$
\left|\left\langle g_{z}, v\right\rangle\right|=\left|\left\langle g_{z}, v-C_{z}\right\rangle\right| \leq\left\|g_{z}\right\|_{L^{p}\left(\omega_{z}\right)}\left\|v-C_{z}\right\|_{L^{q}\left(\omega_{z}\right)} .
$$

Let $\hat{\omega}_{z}:=h_{z}^{-1}\left(\omega_{z}-z\right)$ be the rescaled star, and $\hat{v}(\hat{x}):=v\left(h_{z} \hat{x}+z\right)$ be the corresponding rescaled function. Hence $\left\|v-C_{z}\right\|_{L^{q}\left(\omega_{z}\right)}=h_{z}^{d / q}\left\|\hat{v}-C_{z}\right\|_{L^{q}\left(\hat{\omega}_{z}\right)}$ and

$$
\left\|\hat{v}-C_{z}\right\|_{L^{q}\left(\hat{\omega}_{z}\right)} \lesssim\left\|\hat{v}-C_{z}\right\|_{H_{\hat{\Gamma}}^{s}\left(\hat{\omega}_{z}\right)} .
$$

We now choose the constant $C_{z}$ as the mean value of $\hat{v}$ on $\hat{\omega}_{z}$ and define the operator $\mathcal{L}_{z}$ : $H_{\Gamma}^{s}\left(\omega_{z}\right) \rightarrow H_{\Gamma}^{s}\left(\hat{\omega}_{z}\right)$ to be $\mathcal{L}_{z}(v):=\hat{v}-C_{z}$. For $s=0$ we have

$$
\left\|\mathcal{L}_{z}(v)\right\|_{L^{2}\left(\hat{\omega}_{z}\right)} \leq\|\hat{v}\|_{L^{2}\left(\hat{\omega}_{z}\right)}=h_{z}^{-d / 2}\|v\|_{L^{2}\left(\omega_{z}\right)},
$$

whereas for $s=1$ we use the second Poincaré's inequality

$$
\left\|\mathcal{L}_{z}(v)\right\|_{H^{1}\left(\hat{\omega}_{z}\right)} \lesssim|\hat{v}|_{H^{1}\left(\hat{\omega}_{z}\right)}=h_{z}^{1-d / 2}|v|_{H^{1}\left(\omega_{z}\right)} \leq h_{z}^{1-d / 2}\|v\|_{H^{1}\left(\omega_{z}\right)} .
$$

A space interpolation argument leads to (4.5) because

$$
\left\|\hat{v}-C_{z}\right\|_{H_{\tilde{\Gamma}}^{s}\left(\hat{\omega}_{z}\right)} \lesssim h_{z}^{s-d / 2}\|v\|_{H_{\Gamma}^{s}\left(\omega_{z}\right)} \quad \forall s \in[0,1] .
$$

$2 \partial \omega_{z} \cap \Gamma$ has positive measure. We take $C_{z}=0$ and notice that both (4.6) and (4.7) still holds, the latter because of the first Poincaré's inequality. We thus proceed as in 1 .

Remark 1 (Choice of $p$ ) To apply Lemma 2 and have a finite upper bound, we must choose $p>1$ so that the following conditions are satisfied:

$$
2 s-1<\frac{1}{p}<\frac{s}{d}+\frac{1}{2} .
$$

The right inequality was required in Lemma 2 to have $L^{p} \subset H^{-s}$ on $\omega_{z}$, and the left inequality gives $\mathcal{I} u_{h} \in \mathbb{V}_{h}$ so that the right hand side of (4.5) is finite according to (4.2). For $\rho<3 / 2$ we can pick $p=2$, but for $\rho \geq 3 / 2$ we need to use $p \in(1,2)$. There exists a $p>1$ satisfying (4.8) iff $(2-1 / d) s<3 / 2$, i.e.,

$$
\left(2-\frac{1}{d}\right) \rho<3 .
$$

For $d=1,2$ this condition is always satisfied as $\rho<2$; see Figure 1 for $d=1$. In the case $d=3$, however, the condition is only satisfied for $\rho \in(0,9 / 5)$. 




Fig. 1 Admissible Region of $p(d=1)$ : The gray region indicates admissible points $(p, s)$ with $s=\rho / 2$. For $\rho \in(0,3 / 2)$ we can choose $p=2$; for $\rho \in(3 / 2,2)$ we need to pick some $1<p<2$ in the gray area.

\subsection{A Posteriori Error Estimates}

The following result is a consequence of Lemmas 1, and 2, as well as Remark 1.

Theorem 1 (Upper Bound for Linear Integral Operators) Let $f \in L^{p}(\Omega)$ and $p>1,0<s<1$ satisfy (4.8). We then have the following upper a posteriori error bound

$$
\left\|u-u_{h}\right\|^{2} \lesssim \mathcal{E}^{2}:=\sum_{z \in \mathcal{P}_{h}} \xi_{z}^{2}
$$

where

$$
\xi_{z}:=h_{z}^{s+\frac{d}{2}-\frac{d}{p}}\left\|\left(R-R_{z}\right) \psi_{z}\right\|_{L^{p}\left(\omega_{z}\right)}
$$

and

$$
R_{z}:= \begin{cases}\left\langle R, \psi_{z}\right\rangle /\left\langle 1, \psi_{z}\right\rangle & z \in \mathcal{P}_{h} \cap \Omega \\ 0 & z \in \mathcal{P}_{h} \cap \Gamma\end{cases}
$$

Proof Using Galerkin orthogonality, we easily obtain

$$
\langle R, \varphi\rangle=\sum_{z \in \mathcal{P}_{h}}\left\langle R, \varphi \psi_{z}\right\rangle=\sum_{z \in \mathcal{P}_{h}}\left\langle R,\left(\varphi-\varphi_{z}\right) \psi_{z}\right\rangle
$$

where $\varphi_{z} \in \mathbb{R}$ is any constant. If we choose $\varphi_{z}$ to be the weighted mean value

$$
\varphi_{z}:= \begin{cases}\left\langle\varphi, \psi_{z}\right\rangle /\left\langle 1, \psi_{z}\right\rangle & z \in \mathcal{P}_{h} \cap \Omega \\ 0 & z \in \mathcal{P}_{h} \cap \Gamma\end{cases}
$$

then recalling that of $R_{z}$ is also a weighted mean value yields

$$
\langle R, \varphi\rangle=\sum_{z \in \mathcal{P}_{h}}\left\langle R-R_{z},\left(\varphi-\varphi_{z}\right) \psi_{z}\right\rangle .
$$

If $g_{z}=\left(R-R_{z}\right) \psi_{z}$ for $z \in \mathcal{P}_{h}$, we see that $\int_{\omega_{z}} g_{z}=0$ for $z \in \mathcal{P}_{h} \cap \Omega$. We may thus apply Lemmas 1 and 2 with $p$ satisfying (4.8) to deduce

$$
\|R\|_{*}^{2} \lesssim \sum_{z \in \mathcal{P}_{h} \backslash \mathcal{C}_{h}} h_{z}^{2 s+d(1-2 / p)}\left\|\left(R-R_{z}\right) \psi_{z}\right\|_{L^{p}\left(\omega_{z}\right)}^{2} .
$$

This is the asserted estimate. 
Remark 2 (Convergence Rate of $\mathcal{E}$ ) Theorem 1 only establishes an upper bound and so the question remains whether the effectivity index $\mathcal{E} /\left\|u-u_{h}\right\|$ is of moderate size. This is confirmed in Table 1 in section 8.2 for $s=\frac{1}{2}$.

Remark 3 (Computation of Residual) The function $\mathcal{A} u_{h}$ is in general singular at the element boundaries for $0<s<1$ even though it does not contain Dirac masses. Therefore special care must be exercised in dealing with numerical integration of $\left\|\left(R-R_{z}\right) \psi_{z}\right\|_{L^{p}\left(\omega_{z}\right)}$. Pointwise values of $\mathcal{A} u_{h}(x)$, necessary at quadrature points, can be computed by appropriate quadrature algorithms [50]. We refer to $\S 8$ for more details for $d=1$.

\subsection{Second Order Integro-Differential Operator}

The case C3 of second order integro-differential operators leads to additional jump residual terms. The residual $r_{h}$ contains two parts: a regular part (interior residual) and a singular part (jump residual) with respect to the Lebesgue measure. Let the interior residual associated with $\mathcal{A}$ be

$$
R:=f-\mathcal{I} u_{h}-c_{1} \cdot \nabla u_{h}-c_{0} u_{h},
$$

and the jump residual on the side $\tau_{1} \cap \tau_{2}$ be

$$
J:=-\mu\left(\left.\nabla u_{h}\right|_{\tau_{1}} \cdot \nu_{1}+\left.\nabla u_{h}\right|_{\tau_{2}} \cdot \nu_{2}\right),
$$

where $\nu_{i}$ is the unit outer normal vector to the element $\tau_{i} \in \mathcal{T}_{h}$ for $i=1,2$. In this case, $s=1$, $0<\rho<1$ and, instead of (4.8), the range of admissible $p$ 's becomes

$$
\rho-1<\frac{1}{p}<\frac{1}{d}+\frac{1}{2}
$$

For $\rho<3 / 2$ we may take $p=2$, but we need $p \in[1,2)$ for $\rho>3 / 2$. Such a $p$ exists if $\rho<3 / 2+1 / d$. This condition is verified for $d=1,2$, but it requires $\rho<11 / 6$ for $d=3$.

Theorem 2 (Upper Bound for Integro-differential Operator of Order 2) Let $f \in L^{p}(\Omega)$ and $p>1$ satisfy (4.15). We then have the following upper a posteriori error bound for $s=1$

$$
\left\|u-u_{h}\right\|^{2} \lesssim \mathcal{E}^{2}:=\sum_{z \in \mathcal{P}_{h}}\left(\eta_{z}^{2}+\xi_{z}^{2}\right)
$$

where $\xi_{z}$ is given in (4.10) with $s=1$ and $\eta_{z}$ is the jump residual

$$
\eta_{z}:=h_{z}^{1 / 2}\|J\|_{L^{2}\left(\gamma_{z}\right)} \quad \forall z \in \mathcal{P}_{h} .
$$

Proof Using the partition of unity $\left\{\psi_{z}\right\}_{z \in \mathcal{P}_{h}}$, together with Galerkin orthogonality, we get

$$
\left\langle r_{h}, \varphi\right\rangle=\sum_{z \in \mathcal{P}_{h}}\left\langle r_{h},\left(\varphi-\varphi_{z}\right) \psi_{z}\right\rangle=\sum_{z \in \mathcal{P}_{h}}\left\langle R,\left(\varphi-\varphi_{z}\right) \psi_{z}\right\rangle+\int_{\gamma_{z}} J\left(\varphi-\varphi_{z}\right) \psi_{z} \quad \varphi \in H_{0}^{1}(\Omega),
$$

where $\varphi_{z}$ is given by (4.12) for all $z$. Note that we have integrated elementwise by parts the term $\left\langle\mu \nabla u_{h}, \nabla\left[\left(\varphi-\varphi_{z}\right) \psi_{z}\right]\right\rangle$. The rest of the proof proceeds as that of Theorem 1 for the first term and the standard a posteriori error analysis for the second one.

Remark 4 (Convergence rate of $\mathcal{E}$ ) The definition (4.11) of $R_{z}$ implies that $\left(R-R_{z}\right) \psi_{z}$ has mean value 0 and thus $\xi_{z}$ appears to be an oscillation term. This is confirmed in Table 2 of section 8.2 for $\mathcal{A}=-\Delta+\mathcal{I}$, which shows faster decay of $\left(\sum_{z \in \mathcal{P}_{h}} \xi_{z}^{2}\right)^{\frac{1}{2}}$ than $\left\|u-u_{h}\right\|$. This is in striking contrast with cases $\mathrm{C} 1$ and $\mathrm{C} 2$; see Table 1. 


\section{Elliptic Variational Inequality}

In this section we discuss elliptic variational inequalities of the form (3.3) and (3.5), with the simplifying assumption that the obstacle $\chi$ is piecewise linear in $\mathcal{T}_{h}$. We derive an abstract a posteriori error estimate for integro-differential operators of order smaller than or equal to 2 . We refer to $[60,49,33,48,12]$ for similar analyses for differential operators.

\subsection{Discrete Elliptic Variational Inequalities}

Consider the discrete convex set $\mathcal{K}_{h}$ approximating $\mathcal{K}$

$$
\mathcal{K}_{h}:=\left\{v \in \mathbb{V}_{h}: v \geq \chi\right\}
$$

Since we assume that $\chi$ is piecewise linear over $\mathcal{T}_{h}$, we have $\mathcal{K}_{h} \subset \mathcal{K}$ and the approximation is thus conforming. Property $\mathcal{K}_{h} \subset \mathcal{K}$ greatly simplifies the analysis; we refer to $[45,49,60]$ for general obstacles. We formulate the discrete variational inequality: find $u_{h} \in \mathcal{K}_{h}$ such that

$$
\left\langle\mathcal{A} u_{h}-f, u_{h}-v\right\rangle \leq 0 \quad \forall v \in \mathcal{K}_{h}
$$

\subsection{Lagrange Multipliers and Galerkin Functional}

We define $r_{h}:=f-\mathcal{A} u_{h}$ formally to be the residual as in the case of linear equations. Note that, however, for variational inequalities there is no longer an error-residual relation $\mathcal{A}\left(u-u_{h}\right)=r_{h}$, which is the starting point for residual error estimation. We must account for the constraint.

We first introduce the Lagrange multiplier

$$
\sigma:=f-\mathcal{A} u \in H^{-s}(\Omega)
$$

which restores equality, is non-positive and vanishes in the non-contact region in the sense of distributions. We next introduce a discrete Lagrange multiplier $\sigma_{h}$ and notice the validity of $\mathcal{A}\left(u-u_{h}\right)=r_{h}-\sigma$, or equivalently,

$$
\mathcal{A}\left(u-u_{h}\right)+\sigma-\sigma_{h}=r_{h}-\sigma_{h} .
$$

We discuss practical choices $\sigma_{h}$ so that $\sigma_{h} \leq 0$ in Section 6. Equation (5.4) mimics the errorresidual relation for linear equations. The right-hand side of (5.4) is a nonlinear quantity though, the so-called Galerkin functional,

$$
\mathcal{G}_{h}:=r_{h}-\sigma_{h},
$$

and plays an important role in a posteriori error analysis of variational inequalities. We refer to $[60,33,48,45]$ where this terminology was introduced and used before. 


\subsection{Abstract Error Analysis}

For the moment, we only assume that $\sigma_{h} \leq 0$ and focus on deriving upper and lower error bounds. The structure of these bounds turns out to be independent of particular choices of $\sigma_{h}$.

Multiplying (5.4) by $u-u_{h}$, and recalling (5.5), we obtain via the Cauchy-Schwarz inequality

$$
\left\|u-u_{h}\right\|^{2} \leq \frac{1}{2}\left\|\mathcal{G}_{h}\right\|_{*}^{2}+\frac{1}{2}\left\|u-u_{h}\right\|^{2}-\left\langle\sigma-\sigma_{h}, u-u_{h}\right\rangle
$$

On the other hand, (5.4) also implies

$$
\left\langle\sigma-\sigma_{h}, \varphi\right\rangle=\left\langle\mathcal{A}\left(u_{h}-u\right), \varphi\right\rangle+\left\langle\mathcal{G}_{h}, \varphi\right\rangle \quad \forall \varphi \in \widetilde{H}^{s}(\Omega) .
$$

Therefore $\left\|\sigma-\sigma_{h}\right\|_{*}^{2} \lesssim\left\|u-u_{h}\right\|^{2}+\left\|\mathcal{G}_{h}\right\|_{*}^{2}$, whence we find the upper bound

$$
\left\|u-u_{h}\right\|^{2}+\left\|\sigma-\sigma_{h}\right\|_{*}^{2} \lesssim\left\|\mathcal{G}_{h}\right\|_{*}^{2}-\left\langle\sigma-\sigma_{h}, u-u_{h}\right\rangle .
$$

The conformity assumption $\mathcal{K}_{h} \subset \mathcal{K}$ simplifies the analysis of $\left\langle\sigma-\sigma_{h}, u-u_{h}\right\rangle$; we refer to $[45,49,60]$ for more general cases. In fact, taking $v=u_{h} \in \mathcal{K}$ in (3.3) and using (5.3) yields $\left\langle\sigma, u-u_{h}\right\rangle \geq 0$. Furthermore, since $u \geq \chi$ and $\sigma_{h} \leq 0$, it is easy to see that

$$
\left\langle\sigma_{h}, u-u_{h}\right\rangle=\left\langle\sigma_{h}, u-\chi\right\rangle-\left\langle\sigma_{h}, u_{h}-\chi\right\rangle \leq-\left\langle\sigma_{h}, u_{h}-\chi\right\rangle
$$

whence

$$
\left\|u-u_{h}\right\|^{2}+\left\|\sigma-\sigma_{h}\right\|_{*}^{2} \lesssim\left\|\mathcal{G}_{h}\right\|_{*}^{2}-\left\langle\sigma_{h}, u_{h}-\chi\right\rangle .
$$

Rearranging terms in (5.6), and using the strong sector condition (3.2), we infer that

$$
\left\langle\mathcal{G}_{h}, \varphi\right\rangle=\left\langle\mathcal{A}\left(u-u_{h}\right), \varphi\right\rangle+\left\langle\sigma-\sigma_{h}, \varphi\right\rangle \lesssim\left\|u-u_{h}\right\| \cdot\|\varphi\|+\left\|\sigma-\sigma_{h}\right\|_{*} \cdot\|\varphi\| \quad \forall \varphi \in \widetilde{H}^{s}(\Omega) .
$$

This shows that $\left\|\mathcal{G}_{h}\right\|_{*}^{2}$ is also a lower bound for $\left\|u-u_{h}\right\|^{2}+\left\|\sigma-\sigma_{h}\right\|_{*}^{2}$ up to a multiplicative constant. We summarize the preceding analysis in the following abstract lemma.

Lemma 3 (Abstract Error Bounds for Elliptic Variational Inequalities) Let $u$ and $u_{h}$ be the solutions of (3.3) and (5.2), respectively. If $\sigma_{h} \leq 0$, then we have the upper and lower bounds

$$
\left\|\mathcal{G}_{h}\right\|_{*}^{2} \lesssim\left\|u-u_{h}\right\|^{2}+\left\|\sigma-\sigma_{h}\right\|_{*}^{2} \lesssim\left\|\mathcal{G}_{h}\right\|_{*}^{2}-\left\langle\sigma_{h}, u_{h}-\chi\right\rangle .
$$

Remark 5 (Computable Upper Bound) The estimate (5.8) is of theoretical interest. In practice, we still need to find a localized and computable representation of the global dual norm $\left\|\mathcal{G}_{h}\right\|_{*}^{2}$ and an upper bound for $\left\langle\sigma_{h}, \chi-u_{h}\right\rangle$ as small as possible. This leads to the definition of $\sigma_{h}$ and is our next task in section 6 .

\section{A Localized Residual-type Error Estimator}

In practice, it is important to find a "good" approximation $\sigma_{h}$, which mimics the properties of $\sigma$ at the discrete level. An ideal, but not practical, choice would be $\sigma_{h}=\sigma$. A simple-minded alternative is to take $\sigma_{h}=0$ for which Lemma 3 yields the bounds

$$
\left\|r_{h}\right\|_{*}^{2} \lesssim\left\|u-u_{h}\right\|^{2}+\|\sigma\|_{*}^{2} \lesssim\left\|r_{h}\right\|_{*}^{2} .
$$

However these bounds have the drawback that the residual $r_{h}$ in the contact region contributes to the bound even if $u_{h}$ is the exact solution. This contribution is accounted for in $\|\sigma\|_{*}^{2}$ but overestimates $\left\|u-u_{h}\right\|^{2}$. A good practical estimator should be localized in the sense that only the value of the residual in the non-contact region contributes to the error bound. This is what we construct in this section, thereby extending the work of Fierro and Veeser [33] to integrodifferential operators. 


\subsection{Discrete Sets}

We start by defining the discrete counterparts of the contact set $\mathcal{C}:=\{u=\chi\}$ and non-contact set $\mathcal{N}:=\{u>\chi\}$, which use the sets $\omega_{z}$ and $\gamma_{z}$ for $z \in \mathcal{P}_{h}$.

We recall that the residual $r_{h}=f-\mathcal{A} u_{h}$ contains two parts: a regular part (interior residual $R$ ) and a singular part (jump residual $J$ ) given by (4.13) and (4.14), respectively; the latter vanishes if $s<1$. We split $\mathcal{P}_{h}=\mathcal{N}_{h} \cup \mathcal{C}_{h} \cup \mathcal{F}_{h}$ into three disjoint sets of non-contact nodes $\mathcal{N}_{h}$, full-contact nodes $\mathcal{C}_{h}$, and free boundary nodes $\mathcal{F}_{h}$ defined as follows:

$$
\begin{aligned}
\mathcal{N}_{h} & :=\left\{z \in \mathcal{P}_{h} \mid u_{h}>\chi \text { in int } \omega_{z}\right\}, \\
\mathcal{C}_{h} & :=\left\{z \in \mathcal{P}_{h} \mid u_{h}=\chi \text { and } r_{h} \leq 0 \text { in } \omega_{z}\right\}, \\
\mathcal{F}_{h} & :=\mathcal{P}_{h} \backslash\left(\mathcal{N}_{h} \cup \mathcal{C}_{h}\right)
\end{aligned}
$$

Remark 6 (Sign Condition) Since $r_{h}$ is not a discrete object, it is not possible to check the sign condition $r_{h} \leq 0$ in the definition (6.1b). In practice, we check $R \leq 0$ and $J \leq 0$ at all quadrature points in $\omega_{z}$ and $\gamma_{z}$.

\subsection{Discrete Lagrange Multiplier}

In trying to make $\sigma_{h}$ as close to $r_{h}$ as possible, and thus minimize $\left\|\mathcal{G}_{h}\right\|_{*}$, we first attempt to define $\sigma_{h}$ as a piecewise linear function $\sigma_{h}=\sum_{z \in \mathcal{P}_{h}} s_{z} \psi_{z}$ with nodal values $s_{z}$ given by weighted means of $r_{h}$ on stars $\omega_{z}$ :

$$
s_{z}:= \begin{cases}\left\langle r_{h}, \psi_{z}\right\rangle /\left\langle 1, \psi_{z}\right\rangle & z \in \mathcal{P}_{h} \cap \Omega \\ 0 & z \in \mathcal{P}_{h} \cap \Gamma .\end{cases}
$$

Note that $s_{z}$ can be naturally divided into two parts $s_{z}=R_{z}+J_{z}$, where

$$
R_{z}:=\left\{\begin{array}{ll}
\left\langle R, \psi_{z}\right\rangle /\left\langle 1, \psi_{z}\right\rangle & z \in \mathcal{P}_{h} \cap \Omega \\
0 & z \in \mathcal{P}_{h} \cap \Gamma
\end{array} \quad \text { and } \quad J_{z}:= \begin{cases}-\left\langle\mu \nabla u_{h}, \nabla \psi_{z}\right\rangle /\left\langle 1, \psi_{z}\right\rangle & z \in \mathcal{P}_{h} \cap \Omega \\
0 & z \in \mathcal{P}_{h} \cap \Gamma\end{cases}\right.
$$

and that $s_{z}=0$ on $\Gamma$ is motivated by $\sigma=0$ on $\mathcal{N} \cap \Gamma$. This definition yields $s_{z} \leq 0$ and $s_{z}=0$ for $z \in \mathcal{N}_{h}$, and it is thus quite appropriate for $\mathcal{N}_{h}$ but not necessarily for $z \in \mathcal{C}_{h}$. In fact, to achieve localization of the error estimator, $\sigma_{h}$ must equal the residual $r_{h}$ in $\omega_{z}$ for $z \in \mathcal{C}_{h}$, thereby leading to $\sigma_{h}=r_{h} \leq 0$ in $\omega_{z}$.

We can blend the two competing alternatives via the partition of unity $\left\{\psi_{z}\right\}_{z \in \mathcal{P}_{h}}$ and define

$$
\sigma_{h}:=\sum_{z \in \mathcal{C}_{h}} r_{h} \psi_{z}+\sum_{z \in \mathcal{P}_{h} \backslash \mathcal{C}_{h}} s_{z} \psi_{z},
$$

but $\sigma_{h}$ is not a purely discrete object here [33]. As a consequence of $s_{z} \leq 0$ and the sign conditions in (6.1b), (6.3) guarantees that $\sigma_{h} \leq 0$ in $\Omega$. In addition, the Galerkin functional vanishes in the discrete contact region in the sense of distributions (localization), i.e.

$$
\mathcal{G}_{h}=\sum_{z \in \mathcal{P}_{h}} r_{h} \psi_{z}-\sigma_{h}=\sum_{z \in \mathcal{P}_{h} \backslash \mathcal{C}_{h}}\left(r_{h}-s_{z}\right) \psi_{z}
$$




\subsection{Upper Bound}

In view of Lemma 3 , to derive a practical upper bound of the energy error, we just need to find localized upper bounds for $\left\|\mathcal{G}_{h}\right\|_{*}$ and $\left\langle\sigma_{h}, \chi-u_{h}\right\rangle$. The latter is simple (see Lemma 4) whereas the former is tricky. We first prove that this dual norm can be bounded from above by the sum of local dual norms, and next give computable upper and lower bounds for them.

Error at the Free Boundary. The second term on the right-hand side of (5.8) accounts for lack of monotonicity in the expression (6.3). Proceeding as in [33], [45, Lemma 3.2], and [49], it is easy to see the following assertion.

Lemma 4 (Lack of Monotonicity) If $d_{z}:=\left\langle u_{h}-\chi, \psi_{z}\right\rangle$, then $d_{z} \geq 0$ and the following holds

$$
\left\langle\sigma_{h}, u_{h}-\chi\right\rangle=\sum_{z \in \mathcal{F}_{h}} s_{z} d_{z} .
$$

Proof It suffices to use that $u_{h}=\chi$ in $\omega_{z}$ for any $z \in \mathcal{C}_{h}$ and $s_{z}=0$ for $z \in \mathcal{N}_{h}$.

From Global to Local. Now we are left with $\left\|\mathcal{G}_{h}\right\|_{*}$ or equivalently $\left\|\mathcal{G}_{h}\right\|_{H^{-s}(\Omega)}$. We can now use Lemma 1 to bound the global $H^{-s}(\Omega)$-norm by a sum of localized norms on $\omega_{z}$ for $s \in[0,1]$, and Lemma 2 to obtain a computable estimate in terms of $L^{p}$-norms.

Theorem 3 (Upper Bound for Elliptic Variational Inequalities) Let $f \in L^{p}(\Omega)$ and $p>1$ satisfy (4.8) with $s<1$ or (4.15) with $s=1$. Then the following localized a posteriori upper bound holds

$$
\left\|u-u_{h}\right\|^{2}+\left\|\sigma-\sigma_{h}\right\|_{*}^{2} \lesssim \sum_{z \in \mathcal{P}_{h} \backslash \mathcal{C}_{h}}\left(\eta_{z}^{2}+\xi_{z}^{2}\right)-\sum_{z \in \mathcal{F}_{h}} s_{z} d_{z},
$$

where $s_{z}$ is defined in (6.2), $d_{z}$ in Lemma 4 , and

$$
\eta_{z}:=h_{z}^{\frac{1}{2}}\|J\|_{L^{2}\left(\gamma_{z}\right)} \quad \text { and } \quad \xi_{z}:=h_{z}^{s+\frac{d}{2}-\frac{d}{p}}\left\|\left(R-R_{z}\right) \psi_{z}\right\|_{L^{p}\left(\omega_{z}\right)} .
$$

Proof The expression (6.4) can be written as $\mathcal{G}_{h}=\sum_{z \in \mathcal{P}_{h}} g_{z}$ with $g_{z}=\left(r_{h}-s_{z}\right) \psi_{z}$ for $z \in \mathcal{P}_{h} \backslash \mathcal{C}_{h}$ and $g_{z}=0$ otherwise. Therefore, Lemmas 1 and 2 apply for $s<1$ and give $\left\|\mathcal{G}_{h}\right\|_{*}^{2} \lesssim \sum_{z \in \mathcal{P}_{h} \backslash \mathcal{C}_{h}} \xi_{z}^{2}$. The desired estimate (6.5) follows as in Theorems 1 and 2 (for $s=1$ ) upon using Lemma 4.

\section{Parabolic Problems}

In this section, we consider the parabolic integro-differential equation (1.2) and variational inequality (1.3). For the numerical treatment of the time derivative term, we use the backward Euler approximation because the lack of time regularity of the solution $u(t)$ of $(1.3)$ does not justify higher order schemes.

\subsection{Fully-discrete Problems}

We partition the time domain $[0, T]$ into $N$ subintervals, i.e. $0=t_{0}<t_{1}<\cdots<t_{N}=T$ and let $k_{n}:=t_{n}-t_{n-1}$. For any sequence $\left\{W^{n}\right\}_{n=1}^{N}$, we define the piecewise constant interpolant $\bar{W}$ and the piecewise linear interpolant $W$ to be

$$
\bar{W}(t):=W^{n}, \quad W(t):=l(t) W^{n-1}+(1-l(t)) W^{n} \quad \forall t \in\left(t_{n-1}, t_{n}\right], \quad 1 \leq n \leq N,
$$


where the linear function $l(t)$ is defined by

$$
l(t):=\frac{t_{n}-t}{k_{n}} \quad \forall t \in\left(t_{n-1}, t_{n}\right]
$$

We also denote by $\left\{\delta W^{n}\right\}_{n=1}^{N}$ the discrete derivative of the sequence $\left\{W^{n}\right\}_{n=1}^{N}$

$$
\delta W^{n}:=\frac{W^{n}-W^{n-1}}{k_{n}} \quad \forall 1 \leq n \leq N .
$$

For a function $w$ continuous in time, we let $w^{n}(\cdot):=w\left(t_{n}, \cdot\right)$ be its semi-discrete approximation and $\bar{w}$ be defined as in (7.1). We denote by $U_{h}^{n}$ for $n=0,1, \ldots, N$ the fully discrete solution at time $t_{n}$ for the time-dependent problems.

The fully-discrete numerical method using the backward Euler scheme in time and the continuous linear finite element method in space can be written as follows: starting from $U_{h}^{0}=I_{h} u_{0} \in$ $\mathcal{K}_{h}$, the linear interpolant of $u_{0}$, find $\left\{U_{h}^{n}\right\}_{n=1}^{N} \subset \mathcal{K}_{h}$ such that

$$
\frac{1}{k_{n}}\left\langle U_{h}^{n}-U_{h}^{n-1}, U_{h}^{n}-v\right\rangle+a\left(U_{h}^{n}, U_{h}^{n}-v\right) \leq\left\langle F^{n}, U_{h}^{n}-v\right\rangle \quad \forall v \in \mathcal{K}_{h},
$$

where $F^{n}=f\left(t_{n}\right)$ and $\mathcal{K}_{h}:=\left\{v \in \mathbb{V}_{h}: v \geq \chi\right\}$. To simplify the discussion, we restrict ourselves to the case where the underlying mesh does not change in time and the obstacle $\chi$ is piecewise linear and time-independent. The effect of mesh changes in time is under investigation.

\subsection{Lagrange Multipliers and Galerkin Functional}

We now define the continuous and discrete Lagrange multipliers and Galerkin functional for the parabolic problem (1.3) in analogy to the elliptic case in $\S 6$. Let the Lagrange multiplier $\sigma$ be

$$
\sigma(t):=f(t)-\partial_{t} u(t)-\mathcal{A} u(t) \in H^{-s}(\Omega) \quad \text { a.e. } t \in(0, T),
$$

and note that $\sigma(t)=0$ for (1.2). At each time $t_{n}, n=1, \ldots, N$, we define the residual

$$
r_{h}^{n}:=F^{n}-\delta U_{h}^{n}-\mathcal{A} U_{h}^{n}
$$

and split the set of all nodes $\mathcal{P}_{h}$ into three disjoint sets

$$
\mathcal{P}_{h}=\mathcal{N}_{h}^{n} \cup \mathcal{C}_{h}^{n} \cup \mathcal{F}_{h}^{n},
$$

where non-contact $\mathcal{N}_{h}^{n}$, full-contact $\mathcal{C}_{h}^{n}$, and free boundary $\mathcal{F}_{h}^{n}$ sets are given by (6.1), except that now they may depend on $n$. We next define the discrete Lagrange multiplier $\sigma_{h}^{n}$ as in (6.3) and the discrete Galerkin functional as in (6.4).

\subsection{Coercivity Property}

Now we give a crucial lemma in the error estimation for time-dependent problems; see $[47,45]$.

Lemma 5 (Coercivity) For any $v, w, z \in \mathcal{K}$, the linear integro-differential operator $\mathcal{A}$ satisfies

$$
\langle\mathcal{A} v-\mathcal{A} w, w-z\rangle \leq 2 \gamma^{2}\|v-z\|^{2}-\frac{1}{4}\left(\|v-w\|^{2}+\|z-w\|^{2}\right) .
$$


Proof In view of the strong sector condition (3.2) of $\mathcal{A}$, we get

$$
\begin{aligned}
\langle\mathcal{A} v-\mathcal{A} w, w-z\rangle & =\langle\mathcal{A} v-\mathcal{A} w, w-v\rangle+\langle\mathcal{A} v-\mathcal{A} w, v-z\rangle \\
& \leq-\|v-w\|^{2}+2 \gamma\|v-w\|\|v-z\| \\
& \leq-\frac{1}{2}\|v-w\|^{2}+2 \gamma^{2}\|v-z\|^{2} .
\end{aligned}
$$

In the same way we get $\langle\mathcal{A} v-\mathcal{A} w, w-z\rangle \leq-\frac{1}{2}\|z-w\|^{2}+2 \gamma^{2}\|v-z\|^{2}$. Adding the last two inequalities gives (7.6).

\subsection{Abstract Upper Bound for Parabolic Problems}

Now we are ready to discuss the main steps to treat parabolic problems. Let $\overline{\mathcal{G}}_{h} \in L^{2}\left(0, T ; H^{-s}(\Omega)\right)$ be the piecewise constant (in time) Galerkin functional obtained from $\left\{\mathcal{G}_{h}^{n}\right\}_{n=1}^{N}$, i.e.

$$
\overline{\mathcal{G}}_{h}=\bar{F}-\partial_{t} U_{h}-\mathcal{A} \bar{U}_{h}-\bar{\sigma}_{h} .
$$

This and the definition (7.5) of $\sigma$ give

$$
\left\langle\overline{\mathcal{G}}_{h}, \varphi\right\rangle=\left\langle\mathcal{A}\left(u-\bar{U}_{h}\right), \varphi\right\rangle-\left\langle\partial_{t}\left(u-U_{h}\right)+\left(\sigma-\bar{\sigma}_{h}\right), \varphi\right\rangle-\langle f-\bar{F}, \varphi\rangle \quad \forall \varphi \in \widetilde{H}^{s}(\Omega) .
$$

Taking $\varphi=u-U_{h}$ in (7.7) and applying Lemma 5, we get

$$
\begin{aligned}
& \frac{1}{2} \frac{d}{d t}\left\|u-U_{h}\right\|_{L^{2}(\Omega)}^{2}+\frac{1}{4}\left(\left\|u-\bar{U}_{h}\right\|^{2}+\left\|u-U_{h}\right\|^{2}\right) \\
& \quad \leq 2 \gamma^{2}\left\|\bar{U}_{h}-U_{h}\right\|^{2}-\left\langle\sigma-\bar{\sigma}_{h}, u-U_{h}\right\rangle+\left\langle\overline{\mathcal{G}}_{h}, u-U_{h}\right\rangle+\left\langle f-\bar{F}, u-U_{h}\right\rangle .
\end{aligned}
$$

Since $\left\langle\sigma, u-U_{h}\right\rangle \geq 0, \bar{\sigma}_{h} \leq 0$, and $u \geq \chi$, we obtain that

$$
-\left\langle\sigma-\bar{\sigma}_{h}, u-U_{h}\right\rangle \leq-\left\langle\bar{\sigma}_{h}, U_{h}-\chi\right\rangle .
$$

We then apply Young's inequality with appropriate constants for the last two terms on the right-hand side of (7.8) to get

$$
\begin{aligned}
\frac{1}{2} \frac{d}{d t}\left\|u-U_{h}\right\|_{L^{2}(\Omega)}^{2} & +\frac{1}{4}\left\|u-\bar{U}_{h}\right\|^{2}+\frac{1}{8}\left\|u-U_{h}\right\|^{2} \\
& \leq 2 \gamma^{2}\left\|\bar{U}_{h}-U_{h}\right\|^{2}-\left\langle\bar{\sigma}_{h}, U_{h}-\chi\right\rangle+4\left\|\overline{\mathcal{G}}_{h}\right\|_{*}^{2}+4\|f-\bar{F}\|_{*}^{2} .
\end{aligned}
$$

On the other hand, rearranging terms of (7.7) and applying the strong sector condition (3.2), we have that

$$
\left\|\partial_{t}\left(u-U_{h}\right)+\left(\sigma-\bar{\sigma}_{h}\right)\right\|_{*} \leq 2 \gamma\left\|u-\bar{U}_{h}\right\| \mid\left\|\overline{\mathcal{G}}_{h}\right\|_{*}+\|f-\bar{F}\|_{*} .
$$

Adding (7.9) and (7.10) and dropping all the constants, we get the upper bound:

$$
\begin{aligned}
\frac{d}{d t}\left\|u-U_{h}\right\|_{L^{2}(\Omega)}^{2}+\left\|u-\bar{U}_{h}\right\|^{2} & +\left\|u-U_{h}\right\|^{2}+\left\|\partial_{t}\left(u-U_{h}\right)+\left(\sigma-\bar{\sigma}_{h}\right)\right\|_{*}^{2} \\
& \lesssim\left\|\bar{U}_{h}-U_{h}\right\|^{2}-\left\langle\bar{\sigma}_{h}, U_{h}-\chi\right\rangle+\left\|\overline{\mathcal{G}}_{h}\right\|_{*}^{2}+\|f-\bar{F}\|_{*}^{2} .
\end{aligned}
$$

Integrating in time, we then obtain the following upper bound of the error $e\left(U_{h}, \sigma_{h}\right)^{2}:=\left\|\left(u-U_{h}\right)(T)\right\|_{L^{2}(\Omega)}^{2}+\int_{0}^{T}\left\|u-\bar{U}_{h}\right\|^{2}+\left\|u-U_{h}\right\|^{2}+\left\|\partial_{t}\left(u-U_{h}\right)+\left(\sigma-\bar{\sigma}_{h}\right)\right\|_{*}^{2} d t$. 
Lemma 6 (Abstract Upper Bound for Parabolic Variational Inequalities) Let $u$ and $\left\{U_{h}^{n}\right\}_{n=1}^{N}$ be solutions of the continuous and discrete variational inequalities, (1.3) and (7.4), respectively. Then we have the following upper bound:

$$
\begin{aligned}
e\left(U_{h}, \sigma_{h}\right)^{2} & \lesssim\left\|u_{0}-U_{h}^{0}\right\|_{L^{2}(\Omega)}^{2}+\int_{0}^{T}\left\|\bar{U}_{h}-U_{h}\right\|^{2} d t \\
& +\int_{0}^{T}\left\|\overline{\mathcal{G}}_{h}\right\|_{*}^{2} d t-\int_{0}^{T}\left\langle\bar{\sigma}_{h}, U_{h}-\chi\right\rangle d t+\int_{0}^{T}\|f-\bar{F}\|_{*}^{2} d t .
\end{aligned}
$$

Notice that on the right-hand side of (7.11), the first term measures the initial error; the second term is computable and measures the error due to time discretization; and the last term gives the data consistency error due to time discretization of $f$. The third and fourth terms have been analyzed before for stationary problems and measure the space discretization error. At each time-step, $\left\|\mathcal{G}_{h}^{n}\right\|_{*}$ and $\left\langle\sigma_{h}^{n}, U_{h}-\chi\right\rangle$ can be treated similarly as in $\S 6$.

\subsection{Localized Error Estimators for Parabolic Variational Inequalities}

Finally, we conclude this section by giving a computable local error estimate. Let $R^{n}$ and $J^{n}$ be the interior and jump residuals at time $t_{n}$, respectively, i.e.

$$
R^{n}:=F^{n}-\delta U_{h}^{n}-\mathcal{I} U_{h}^{n}-c_{1} \cdot \nabla U_{h}^{n}-c_{0} U_{h}^{n} \quad \text { and } \quad J^{n}:=-\mu\left(\left.\nabla U_{h}^{n}\right|_{\tau_{1}} \cdot \nu_{1}+\left.\nabla U_{h}^{n}\right|_{\tau_{2}} \cdot \nu_{2}\right),
$$

the latter used for $s=1$ only. We define the following interior and jump indicators as in $\S 6$ :

$$
\eta_{z}^{n}:=h_{z}^{\frac{1}{2}}\left\|J^{n}\right\|_{L^{2}\left(\gamma_{z}\right)} \quad \text { and } \quad \xi_{z}^{n}:=h_{z}^{s+\frac{d}{2}-\frac{d}{p}}\left\|\left(R^{n}-R_{z}^{n}\right) \psi_{z}\right\|_{L^{p}\left(\omega_{z}\right)},
$$

where $R_{z}^{n}:=\left\langle R^{n}, \psi_{z}\right\rangle /\left\langle 1, \psi_{z}\right\rangle$ is the weighted average. We define the error estimator to be

$$
\mathcal{E}:=\left(\mathcal{E}_{0}^{2}+\mathcal{E}_{k}^{2}+\mathcal{E}_{h}^{2}+\mathcal{E}_{D}^{2}\right)^{\frac{1}{2}}
$$

with

$$
\begin{array}{lrl}
\mathcal{E}_{0}^{2} & =\left\|u_{0}-U_{h}^{0}\right\|_{L^{2}(\Omega)}^{2} & \text { initial error } \\
\mathcal{E}_{k}^{2} & =\sum_{n=1}^{N} k_{n}\left\|U_{h}^{n}-U_{h}^{n-1}\right\|^{2} d t & \text { time error } \\
\mathcal{E}_{h}^{2}=\sum_{n=1}^{N} k_{n}\left\{\sum_{z \in \mathcal{P}_{h} \backslash \mathcal{C}_{h}^{n}}\left[\left(\eta_{z}^{n}\right)^{2}+\left(\xi_{z}^{n}\right)^{2}\right]-\sum_{z \in \mathcal{F}_{h}^{n}} s_{z}^{n}\left\langle U_{h}^{n-1}-\chi, \psi_{z}\right\rangle\right\} & \text { space error } \\
\mathcal{E}_{D}^{2}=\int_{0}^{T}\|f-\bar{F}\|_{*}^{2} d t . & \text { data oscillation }
\end{array}
$$

We then obtain the following computable and localized upper a posteriori error bound.

Theorem 4 (Upper Bound for Parabolic Variational Inequalities) Let $f \in L^{2}\left(0, T ; L^{p}(\Omega)\right)$ and $p>1,0<\rho<2,0<s \leq 1$ satisfy

$$
\rho-1<\frac{1}{p}<\frac{s}{d}+\frac{1}{2} .
$$

Then the localized upper bound for the error $e\left(U_{h}, \sigma_{h}\right) \lesssim \mathcal{E}$ is valid.

Proof In view of Lemma 6 , it suffices to apply Lemmas 1 and 2 to $\left\|\mathcal{G}_{h}^{n}\right\|_{*}$ and Lemma 4 to $\left\langle\sigma_{h}^{n}, U_{h}^{n}-\chi\right\rangle$. 


\section{Numerical Experiments}

In this section we illustrate the behavior of the proposed error estimators with numerical experiments. Our original motivation was the pricing of American options on a single asset with possible barriers. In this case the singularities which reduce the convergence rates are located at

- the barriers (endpoints of the interval)

- the free boundary

- $t=0$ (maturity) where the payoff function (initial condition) is nonsmooth

and we show the performance of the estimator under these circumstances. These issues also occur in the equality case and the time independent case where we can observe their effect more easily.

We have proved that the estimators give an upper bound to the actual error. With the following numerical experiments we provide additional evidence that

- the space and time estimators converge with the correct rates in space and time

- the ratio of estimator to actual error (effectivity index) is of moderate size

- the node-based error estimators provide reliable information about the local errors

- in the time-independent case an adaptive algorithm driven by our estimators converges with the optimal rate despite the presence of singularities at boundaries and the free boundary.

We consider $d=1, \Omega=(-1,1)$ and the integral operator $\mathcal{I}: \widetilde{H}^{s}(\Omega) \rightarrow H^{-s}(\Omega)$ given by (3.7)-(3.9) with

$$
K(x):=\frac{1}{|x|^{1+\rho}}, \quad x \neq 0 .
$$

with the order $\rho \in\left(0, \frac{3}{2}\right)$. In this case we can use $p=2$ in (4.10) and let

$$
\mathcal{E}_{\eta}^{2}:=\sum_{z \in \mathcal{P}_{h} \backslash \mathcal{C}_{h}} \eta_{z}^{2} \quad \text { and } \quad \mathcal{E}_{\xi}^{2}:=\sum_{z \in \mathcal{P}_{h} \backslash \mathcal{C}_{h}} \xi_{z}^{2} .
$$

We decompose $\Omega$ into $M$ subintervals and therefore have DOF $:=M-1$ degrees of freedom.

\subsection{Implementation Details}

Before discussing the numerical experiments, we first give a few comments on the implementation of the finite element method and of the error estimators. We use mesh points $-1=x_{0}<x_{1}<$ $\cdots<x_{M}=1$. We first consider a uniform mesh with $h_{i}:=x_{i}-x_{i-1}=2 / M$. In $\S 8.3$ we define a graded mesh which has smaller subintervals near the endpoints.

The elements of the stiffness matrix can be expressed analytically in terms of a fourth antiderivative of the kernel function $K(x)$. In this way we can evaluate the stiffness matrix without quadrature error.

The error estimator $\xi_{z}$ in (4.10) is an integral over two adjacent intervals involving the residual $R$. Since a functions $u_{h} \in \mathbb{V}_{h}$ has discontinuous derivatives at the mesh points, the term $\mathcal{I} u_{h}$ will have singularities at the mesh points. If $\rho$ is noninteger these singularities are of the type $\left|x-x_{j}\right|^{1-\rho}$, whereas in the integer case there are logarithmic terms. We used the following quadrature method which gives exponential convergence in the presence of these singularities. Let $-1=x_{0}<x_{1}<\cdots<x_{M}=1$ be the mesh points of $\Omega$. Since the residual $r$ is singular at the ends of each interval, we subdivide $\left[x_{i-1}, x_{i}\right]$ of length $h_{i}$ in the following way: Let $P>0$ be an integer and $\theta=0.1$. We introduce additional points at distance $\theta^{j} h_{i}$ from the left and right 
endpoints, for $j=1, \ldots, P$. This divides the interval into $1+2 P$ subintervals. On each of these intervals a $Q$-point Gauss-Legendre rule is applied for numerical integration. Also the condition $r \leq 0$ in the definition of discrete contact set $\mathcal{C}_{h}$ is checked pointwise at each of the $(1+2 P) Q$ quadrature points. It is shown in [53] that the quadrature error decrease exponentially fast with respect to $P Q$.

In all our numerical computations we used three subintervals $(P=1)$ with two Gauss quadrature points in each $(Q=2)$. We performed numerical experiments which indicated that increasing $P$ and $Q$ does not change the character of the asymptotic results.

The linear complementarity problem (5.2) can be solved by the projected SOR method [26], multilevel methods [38,57], domain decomposition methods [6], and interior point methods [8]; see [35] and references therein. We used the projected SOR method in our simulations.

\subsection{Elliptic Equations}

In this example, we consider problem (4.1) with $\rho=1$. To test the asymptotic behavior of the error estimators, we construct a problem where the exact solution is available. We define the effectivity index to be the ratio $\mathcal{E} /\left\|u-u_{h}\right\|$.

Pure Integral Operator Case. We consider case C1 with $c_{0}=c_{1}=0$, then $s=\frac{1}{2}$ and the energy space is $\widetilde{H}^{\frac{1}{2}}(\Omega)$. We choose $f(x)=\frac{15}{8}-\frac{15}{2} x^{2}+5 x^{4}$, then the exact solution for this problem is $u=\frac{1}{\pi}\left(1-x^{2}\right)^{5 / 2}$. The exact solution $u$ is sufficiently smooth at the boundary $x= \pm 1$ so that the approximation rate in the energy norm is $\mathrm{DOF}^{-1.5}$. The numerical experiment (see Table 1) shows that both the energy error and the error estimator $\mathcal{E}_{\xi}(\eta=0)$ converge with this optimal rate 1.5. Furthermore, the effectivity index is almost a constant (around 5.0).

Although we only prove reliability of the proposed error estimator $\mathcal{E}$, we notice that the nodebased error indicator $\xi_{z}$ mimics the pointwise error (see Figure 2). This observation justifies the use of $\left\{\xi_{z}\right\}_{z \in \mathcal{P}_{h} \backslash \mathcal{C}_{h}}$ to drive adaptive algorithms.

\begin{tabular}{|c|c|c|c|}
\hline DOF & $\left\|u-u_{h}\right\|$ & $\mathcal{E}$ & Effectivity \\
\hline \hline 15 & $1.3021 \mathrm{e}-002$ & $6.2052 \mathrm{e}-002$ & 4.7655 \\
31 & $4.4597 \mathrm{e}-003$ & $2.2014 \mathrm{e}-002$ & 4.9362 \\
63 & $1.5618 \mathrm{e}-003$ & $7.7849 \mathrm{e}-003$ & 4.9846 \\
127 & $5.5069 \mathrm{e}-004$ & $2.7527 \mathrm{e}-003$ & 4.9986 \\
255 & $1.9455 \mathrm{e}-004$ & $9.7327 \mathrm{e}-004$ & 5.0027 \\
\hline EOC & 1.501 & 1.500 & - \\
\hline
\end{tabular}

Table 1 Elliptic equation in $\S 8.2$ with pure integral operator $\mathcal{A}=\mathcal{I}$ of order $2 s=\rho=1$ (uniform mesh, expected convergence rate 1.5). EOC is the experimental convergence rate based on last two iterations, which agrees with the expected value 1.5. The effectivity index (ratio between the error estimator and the error) is almost constant.

Integro-Differential Operator Case. Now we consider case C3 and take $\mathcal{A}=-\Delta+\mathcal{I}$ with $\rho=s=1$, then the energy space is $\widetilde{H}^{1}(\Omega)$. We choose an appropriate right-hand side function $f$ such that the exact solution is exactly the same as in the previous example. The energy norm error as well as the error estimators are reported in Table 2. We see that the jump residual term $\eta$ converges at the optimal convergence rate $\left(\mathrm{DOF}^{-1.0}\right)$ just as the energy error itself. On the other hand, $\mathcal{E}_{\xi}$ is of higher order as we expected (see Remark 4). As in the last example, Figure 3 shows the nodal-based error indicator captures the local behavior of the pointwise error. 

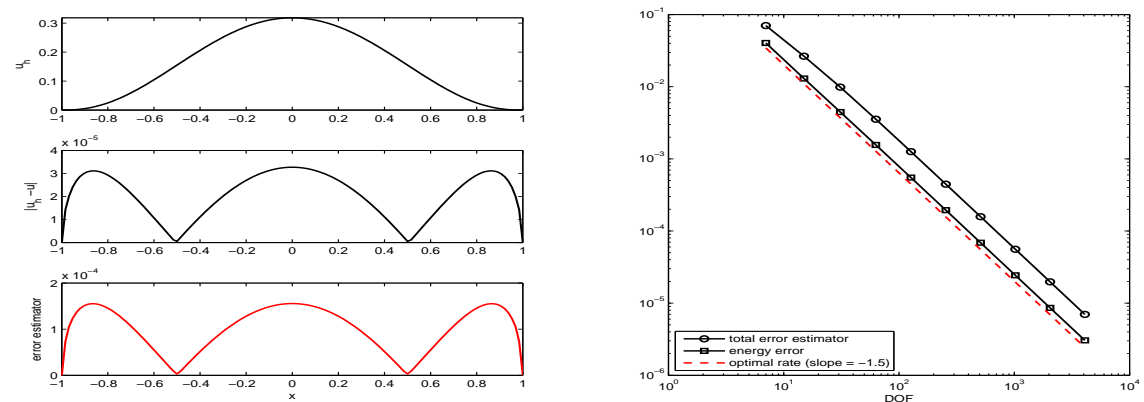

Fig. 2 Elliptic equation in $\$ 8.2$ with pure integral operator $\mathcal{A}=\mathcal{I}$ of order $2 s=\rho=1$ (uniform mesh). Upper left: numerical solution; middle left: pointwise error $\left|u_{h}-u\right|$; lower left: nodal-based error estimator; right: convergence rates for energy error and estimator $\mathcal{E}$. Both the energy error and the error estimator converge at the optimal rate $O\left(\mathrm{DOF}^{-1.5}\right)$. Furthermore, the local error indicator captures the local behavior of the error.

\begin{tabular}{|c|c|c|c|c|c|}
\hline DOF & $\left\|u-u_{h}\right\|$ & $\mathcal{E}_{\eta}$ & $\mathcal{E}_{\xi}$ & $\mathcal{E}$ & Effectivity of $\mathcal{E}$ \\
\hline \hline 15 & $5.9891 \mathrm{e}-002$ & $1.9670 \mathrm{e}-001$ & $9.6446 \mathrm{e}-003$ & $1.9694 \mathrm{e}-001$ & 3.2883 \\
31 & $2.9484 \mathrm{e}-002$ & $1.0010 \mathrm{e}-001$ & $2.4849 \mathrm{e}-003$ & $1.0013 \mathrm{e}-001$ & 3.3962 \\
63 & $1.4647 \mathrm{e}-002$ & $5.0323 \mathrm{e}-002$ & $6.2787 \mathrm{e}-004$ & $5.0327 \mathrm{e}-002$ & 3.4361 \\
127 & $7.3015 \mathrm{e}-003$ & $2.5203 \mathrm{e}-002$ & $1.5751 \mathrm{e}-004$ & $2.5204 \mathrm{e}-002$ & 3.4519 \\
255 & $3.6455 \mathrm{e}-003$ & $1.2608 \mathrm{e}-002$ & $3.9419 \mathrm{e}-005$ & $1.2608 \mathrm{e}-002$ & 3.4585 \\
\hline EOC & 1.002 & 0.999 & 1.998 & 0.999 & - \\
\hline
\end{tabular}

Table 2 Elliptic equation in $\S 8.2$ with integro-differential operator $\mathcal{A}=-\Delta+\mathcal{I}, s=\rho=1$ (uniform mesh, expected convergence rate 1.0). The experimental convergence rate EOC, based on last two iterations, agrees with the expected value 1.0.
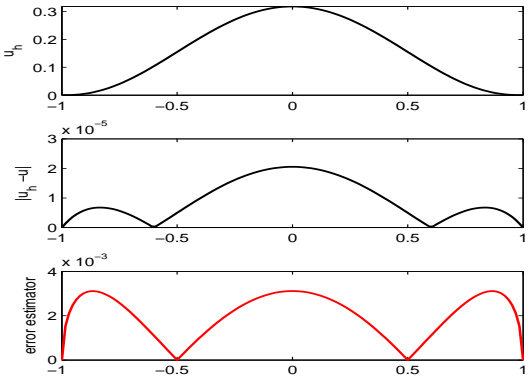

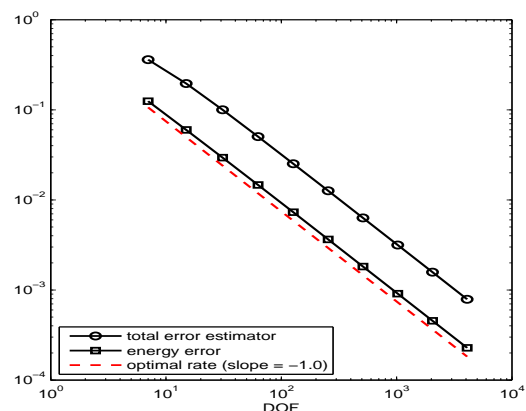

Fig. 3 Elliptic equation in $\$ 8.2$ with integro-differential operator $\mathcal{A}=-\Delta+\mathcal{I}, s=\rho=1$ (uniform mesh). Upper left: numerical solution; middle left: pointwise error; lower left: nodal-based error estimator; right: convergence rates for energy error and estimator $\mathcal{E}$ (optimal convergence rate is $\left.O\left(\mathrm{DOF}^{-1}\right)\right)$.

\subsection{Elliptic Variational Inequalities}

In order to exhibit the influence of the singular behavior of the solution at the free boundary points we now consider $\mathcal{A}$ as in case $\mathrm{C} 1$ with $\rho=0.2$ and $c_{0}=c_{1}=0$, then the energy space is $\widetilde{H}^{s}(\Omega)=H_{0}^{1}(\Omega)$ with $s=0.1$. We consider the problem $(3.3)$ with $f=0$ and the obstacle

$$
\chi(x)=\max (0.5-|x|, 0) .
$$


For a smooth function $u$, we would have an approximation rate $\inf _{v_{h} \in \mathbb{V}_{h}}\left\|u-v_{h}\right\|=O\left(\mathrm{DOF}^{-1.9}\right)$ using uniform mesh refinement.

In our case the solution has singular behavior at the boundary points $x= \pm 1$, and at the free boundary points. The solution of the integral equation $\mathcal{I} u=f$ behaves near the boundary point 1 like $|x-1|^{s}[10$, Theorem 7.5]. We compensate the singular behavior at the boundary points by using mesh grading towards $x=1$. We briefly review the arguments from [62].

In order to analyze the convergence rate we consider the singular term $u(x)=x^{s}$ and use a graded mesh with mesh points $x_{j}=\left(\frac{j}{M}\right)^{\beta}$ for $j=0,1, \ldots, M$. Let $h_{j}$ be the length of subinterval $\Omega_{j}:=\left[x_{j-1}, x_{j}\right]$ and $\Omega:=\cup_{j=1}^{M} \Omega_{j}$. Then we have

$$
h_{j}=x_{j}-x_{j-1} \leq C_{\beta}\left(\frac{j-1}{M}\right)^{\beta-1} \frac{1}{M} \leq C_{\beta} x^{\frac{\beta-1}{\beta}} \frac{1}{M} .
$$

Let $u_{I}$ denote the piecewise linear Lagrange interpolation of $u$. Note that $u-u_{I}$ is zero at all mesh points $x_{j}$. Hence the restriction of $u-u_{I}$ to $\Omega_{j}$ is contained in $\widetilde{H}^{s}\left(\Omega_{j}\right)$. Any function $v \in \widetilde{H}^{s}(\Omega)$ whose restriction $v_{j}$ to each $\Omega_{j}$ is in $\widetilde{H}^{s}\left(\Omega_{j}\right)\left(v_{j}(x)=v(x)\right.$ if $x \in \Omega_{j}$ and $v_{j}(x)=0$ elsewhere) satisfies that $\|v\|_{\widetilde{H}^{s}(\Omega)}^{2} \leq \sum_{j=1}^{M}\|v\|_{\widetilde{H}^{s}\left(\Omega_{j}\right)}^{2}$. This is obvious for $s=0,1$ and follows for $s \in(0,1)$ by interpolation argument. For $j=2, \ldots, M$, we have

$$
\left\|u-u_{I}\right\|_{\widetilde{H}^{s}\left(\Omega_{j}\right)}^{2} \lesssim h_{j}^{4-2 s}|u|_{H^{2}\left(\Omega_{j}\right)}^{2} \lesssim M^{-(4-2 s)} \int_{x_{j-1}}^{x_{j}} x^{-(4-2 s) / \beta} d x,
$$

whereas for $j=1$ we get

$$
\left\|u-u_{I}\right\|_{\widetilde{H}^{s}\left(\Omega_{1}\right)}^{2} \leq \int_{0}^{x_{1}} 1 d y \leq M^{-\beta} .
$$

Hence we obtain for the sum of the squares of the interpolation errors:

$$
\sum_{j=2}^{M}\left\|u-u_{I}\right\|_{\widetilde{H}^{s}\left(\Omega_{j}\right)}^{2} \lesssim M^{-(4-2 s)} \int_{x_{1}}^{1} x^{-(4-2 s) / \beta} d x .
$$

We can see that if $\beta>4-2 s$ then the approximation error in $\widetilde{H}^{s}(\Omega)$-norm converges with the optimal rate $M^{-(2-s)}$ despite the singularity at 0 . So we use the graded mesh $1-\left(\frac{j}{M}\right)^{4}$ for $j=0,1, \ldots, M$ toward the end point 1 in this test example. Because of symmetry, the same grading is employed close to -1 . Note that if the grading parameter $\beta=4$ or a stronger one is used, the smallest meshsize is $M^{-\beta}$ and it decreases very quickly as the degrees of freedom $\mathrm{DOF}=M-1$ increases. Round-off errors will play an important role even for not so big DOF.

According to [55] the solution has regularity $C^{1, s}$ near the free boundary. If the singularity has the form $\left|x-x_{F}\right|^{\gamma}$ near a free boundary point $x_{F}$, then this result would correspond to $\gamma=1+s, u(x) \in H^{1.5+s-\varepsilon}(\Omega)$, and an approximation rate $O\left(\mathrm{DOF}^{-1.5+\varepsilon}\right)$ in the $\widetilde{H}^{s}(\Omega)$ energy norm. Figure 4 shows that we indeed obtain the suboptimal rate of $O\left(\mathrm{DOF}^{-1.5}\right)$ for the error estimator.

In order to obtain the optimal rate $O\left(\mathrm{DOF}^{-1.9}\right)$ we need mesh refinement close to the free boundary whose position we do not know in advance. Therefore we use an adaptive algorithm with the standard estimate - mark - refine strategy (see, e.g., [52]) driven by our local error indicator $\xi_{z}$. Figure 5 shows that the estimator, and therefore the solution, converges with the optimal rate $O\left(\mathrm{DOF}^{-1.9}\right)$. Note that this requires that the algorithm generates appropriate mesh refinement both at the endpoints and at the free boundary points. 

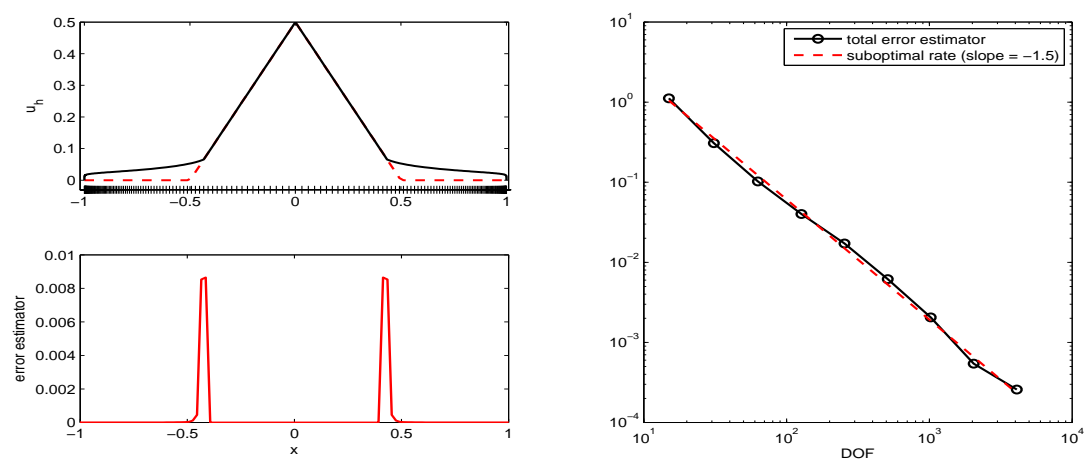

Fig. 4 Elliptic variational inequality in $\S 8.3$ for pure integral operator $\mathcal{A}=\mathcal{I}$ of order $2 s=\rho=0.2$ (using algebraically graded mesh towards end points). Upper left: numerical solution (black solid) and obstacle (red dashed) on the graded mesh with 255 grid points; lower left: nodal-based error estimator in logarithmic scale $\log \left(\xi_{z}\right)$; right: the convergence rate of error estimator is suboptimal $(-1.5$ instead of -1.9$)$ due to the singularity at the free boundary. The proposed local error indicator captures the local behavior of the error and the convergence rate of the error estimator behaves as expected.
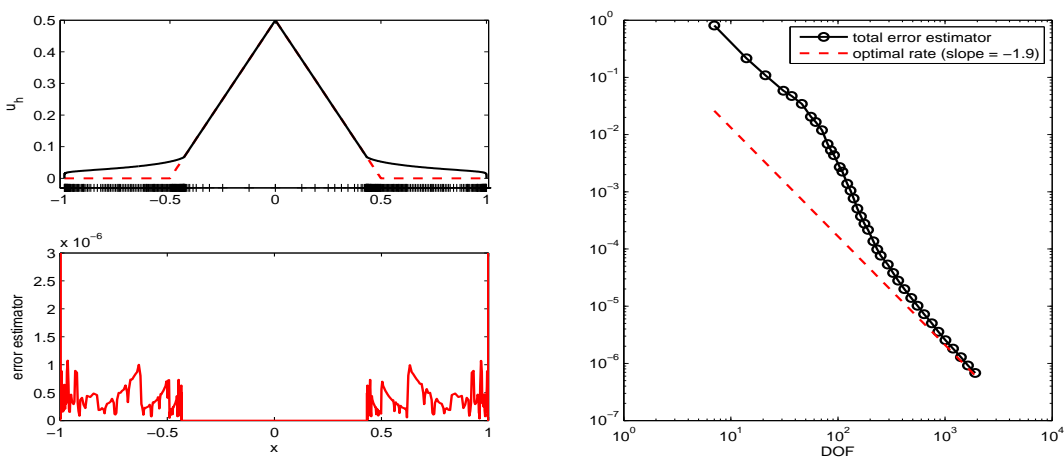

Fig. 5 Elliptic variational inequality in $\S 8.3$ for pure integral operator $\mathcal{A}=\mathcal{I}$ of order $2 s=\rho=0.2$ (adaptive refinement). Upper left: numerical solution (black solid), obstacle (red dashed), and associated mesh points; lower left: nodal-based error estimator in $\operatorname{logarithmic}$ scale $\log \left(\xi_{z}\right)$; right: convergence rate (compare with Figure 4). The adaptive algorithm achieves the optimal convergence rate -1.9 .

\subsection{Parabolic Variational Inequalities}

In this example, we examine the parabolic variational inequality (3.4) with $\mathcal{A}$ as in case $\mathrm{C} 1$ with $\rho=2 s=1$ and $c_{0}=c_{1}=0$. To mimic an American-style butterfly option we use $f=0$, $\chi(x)=\max \left(\frac{1}{2}-|x|, 0\right)$, and $u_{0}=\chi$. We compute the solution in the time interval $[0, T]$ with $T=1$. The solution as well as space error estimator at the two times $t=0.0625$ and $t=0.5$ are shown in Figure 6. We use for the space discretization a mesh of $M$ subintervals with grading toward $x= \pm 1$ to compensate for the singularities at the boundary points. For example, the graded mesh points toward 1 are $1-\left(\frac{j}{M}\right)^{\beta}$ for $j=0,1, \ldots, M$ with $\beta=3$. The choice of $\beta$ is explained in the previous subsection $\$ 8.3$. For the time discretization we use a uniform mesh of $N$ subintervals of size $k=1 / N$. We want to investigate the influence of $M$ and $N$ on the 

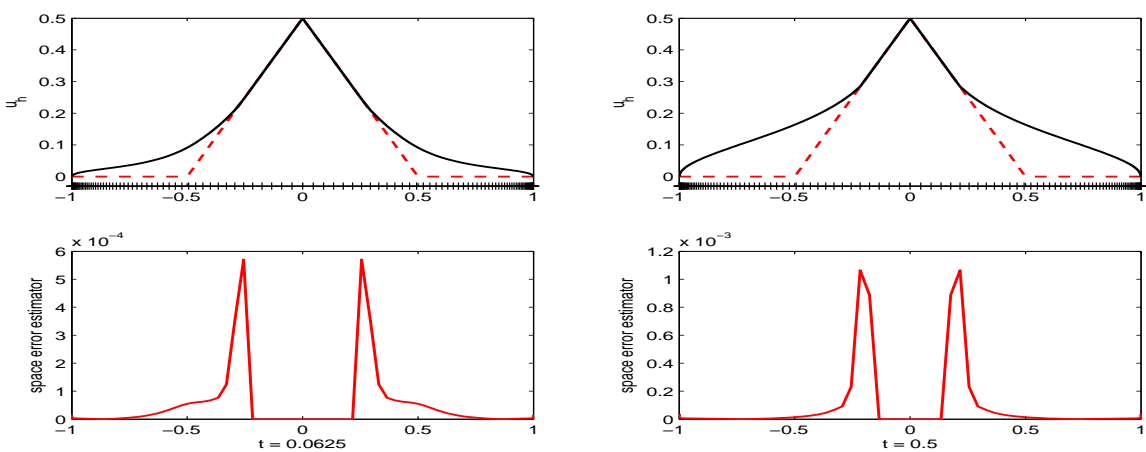

Fig. 6 Parabolic variational inequality in $\S 8.4$ for pure integral operator of order $2 s=\rho=1$ (using algebraically graded mesh in space and uniform in time). Numerical solution and the corresponding space error indicator which captures the correct local space behavior of the solution $(N=128$ and DOF $=127)$.

$L^{2}\left(0, T ; \widetilde{H}^{s}(\Omega)\right)$ error, and how it is reflected by the space error estimator $\mathcal{E}_{h}$ and the time error estimator $\mathcal{E}_{k}$ in $(7.12)$.

In order to understand the effect of the space and time discretization, we consider the parabolic equation $u_{t}+\mathcal{A} u=0$. It is known, even for non-symmetric operator $\mathcal{A}$, that for $s_{2}>s_{1}[34,59]$

$$
u_{0} \in \widetilde{H}^{s_{1}}(\Omega) \Longrightarrow\|u(t)\|_{\widetilde{H}^{s_{2}(\Omega)}} \lesssim t^{-\left(s_{2}-s_{1}\right) / 2}\left\|u_{0}\right\|_{\widetilde{H}^{s_{1}}(\Omega)} .
$$

We examine first a (space) semidiscrete problem which is continuous in time. We ignore the effect of the singularities at the interval boundaries (they are compensated by the mesh grading). The energy error $\left\|u(\cdot, t)-u_{h}(\cdot, t)\right\|$ at time $t$ has to be at least as large as the approximation error $\inf _{v_{h} \in \mathbb{V}_{h}}\left\|u(\cdot, t)-v_{h}\right\| \lesssim h^{2-s}\|u(\cdot, t)\|_{H^{2}(\Omega)}$ according to (8.2). Because of $u_{0} \in H^{\frac{3}{2}-\varepsilon}(\Omega)$ we expect $\left\|u(\cdot, t)-u_{h}(\cdot, t)\right\| \lesssim h^{2-s} t^{-\frac{1}{4}-\varepsilon}$ and an $L^{2}\left(0, T ; \widetilde{H}^{s}(\Omega)\right)$ error of optimal order $h^{\frac{3}{2}}$, despite the presence of a singularity at $t=0$.

We consider finally a (time) semidiscrete problem which is continuous in space. Since $u_{0} \in$ $\widetilde{H}^{\frac{3}{2}-\varepsilon}(\Omega)$, the initial condition $u_{0}$ is in the domain of $\mathcal{A}$, namely $\widetilde{H}^{1}(\Omega)$. Therefore, [47] yields an optimal rate $O(k)$ for the $L^{2}\left(0, T ; \widetilde{H}^{\frac{1}{2}}(\Omega)\right)$-error.

For the numerical computation we used the same number of time and space degrees of freedom, i.e. $N=$ DOF. The left picture of Figure 7 confirms that the time estimator $\mathcal{E}_{k}$ converges with the optimal rate $O\left(N^{-1}\right)$. The right graph of Figure 7 shows that the space estimator $\mathcal{E}_{h}$ also converges with the optimal rate $O\left(\mathrm{DOF}^{-1.5}\right)$, as expected.

\section{Acknowledgment}

We are grateful to Professor Christoph Schwab for valuable discussions on integro-differential operators and their mapping properties, in particular the regularity of $\mathcal{A} u_{h}$ in $\S 4$. We would also like to thank the referees for their critical comments and suggestions that led to an improved presentation of our results. 

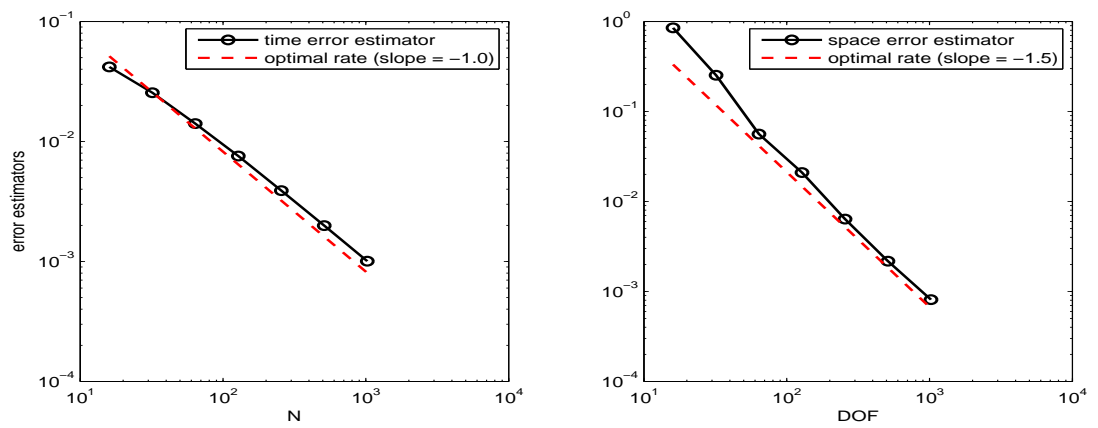

Fig. 7 Parabolic variational inequality in $\$ 8.4$ for pure integral operator of order $2 s=\rho=1$ (using algebraically graded mesh in space and uniform in time). Asymptotic convergence rates of the error estimators, $\mathcal{E}_{k}$ in time (left) and $\mathcal{E}_{h}$ in space (right). Both temporal and spatial convergence rates are optimal. The error estimators behave exactly as the error asymptotically which confirms our theoretical results.

\section{References}

1. M. Ainsworth and J. T. Oden. A posteriori error estimation in finite element analysis. Pure and Applied Mathematics (New York). John Wiley \& Sons, New York, 2000.

2. A. Almendral. Numerical valuation of American options under the CGMY process. In Exotic option pricing and advanced Lévy models, pages 259-276. Wiley, Chichester, 2005.

3. A. Almendral and C. W. Oosterlee. Accurate evaluation of European and American options under the CGMY process. SIAM J. Sci. Comput., 29(1):93-117 (electronic), 2007.

4. A. Almendral and C. W. Oosterlee. On American options under the variance gamma process. Appl. Math. Finance, 14(2):131-152, 2007.

5. R. Araya, E. Behrens, and R. Rodriguez. A posteriori error estimates for elliptic problems with Dirac delta source terms. Numer. Math., 105:193-216, 2006.

6. L. Badea and J. Wang. An additive Schwarz method for variational inequalities. Math. Comp., 69(232):1341-1354, 2000.

7. C. Baiocchi. Discretization of evolution variational inequalities. In Partial differential equations and the calculus of variations, Vol. I, volume 1 of Progr. Nonlinear Differential Equations Appl., pages 59-92, Boston, MA, 1989. Birkhäuser Boston.

8. R. E. Bank, P. E. Gill, and R. F. Marcia. Interior methods for a class of elliptic variational inequalities. In Large-scale PDE-constrained optimization (Santa Fe, NM, 2001), volume 30 of Lect. Notes Comput. Sci. Eng., pages 218-235. Springer, Berlin, 2003.

9. J. Bergh and J. Löfström. Interpolation Spaces. Springer, 1976.

10. S. I. Boyarchenko and S. Z. Levendorskiǔ. Non-Gaussian Merton-Black-Scholes theory, volume 9 of Advanced Series on Statistical Science 83 Applied Probability. World Scientific Publishing Co. Inc., River Edge, NJ, 2002.

11. S. I. Boyarchenko and S. Z. Levendorskii. Perpetual American options under Lévy processes. SIAM J. Control Optim., 40(6):1663-1696, 2002.

12. D. Braess. A posteriori error estimators for obstacle problems-another look. Numer. Math., 101(3):415-421, 2005.

13. H. Brézis. Opérateurs maximaux monotones et semi-groupes de contractions dans les espaces de Hilbert. North-Holland Publishing Co., Amsterdam, 1973. North-Holland Mathematics Studies, No. 5. Notas de Matemática (50).

14. L. Caffarelli and L. Silvestre. Regularity theory for fully nonlinear integro-differential equations. arXiv.org:0709.4681, 2007.

15. L. Caffarelli and A. Vasseur. Drift diffusion equations with fractional diffusion and the quasigeostrophic equation. arXiv.org:math/0608447, 2006.

16. P. Carr, H. Geman, D. B. Madan, and M. Yor. The fine structure of asset returns: An empirical investigation. Journal of Business, 75:305-332, 2002.

17. P. Carr, H. Geman, D. B. Madan, and M. Yor. Stochastic volatility for Lévy processes. Math. Finance, 13(3):345-382, 2003. 
18. C. Carstensen. Efficiency of a posteriori BEM-error estimates for first-kind integral equations on quasi-uniform meshes. Math. Comp., 65(213):69-84, 1996.

19. C. Carstensen. An a posteriori error estimate for a first-kind integral equation. Math. Comp., 66(217):139-155, 1997.

20. C. Carstensen and E. P. Stephan. A posteriori error estimates for boundary element methods. Math. Comp., 64(210):483-500, 1995.

21. Z. Chen and J. Feng. An adaptive finite element algorithm with reliable and efficient error control for linear parabolic problems. Math. Comp., 73(247):1167-1193 (electronic), 2004.

22. S. S. Clift and P. A. Forsyth. Numerical solution of two asset jump diffusion models for option valuation. Appl. Numer. Math., 58(6):743-782, 2008.

23. P. Constantin. Euler equations, Navier-Stokes equations and turbulence. In Mathematical foundation of turbulent viscous flows, volume 1871 of Lecture Notes in Math., pages 1-43. Springer, Berlin, 2006.

24. R. Cont and P. Tankov. Financial modelling with jump processes. Chapman \& Hall/CRC Financial Mathematics Series. Chapman \& Hall/CRC, Boca Raton, FL, 2004.

25. M. Costabel and W. L. Wendland. Strong ellipticity of boundary integral operators. J. Reine Angew. Math., 372:34-63, 1986.

26. C. W. Cryer. Successive overrelaxation methods for solving linear complementarity problems arising from free boundary problems, pages 109-131. Ist. Naz. Alta Mat. Francesco Severi, Rome, 1980.

27. Y. d'Halluin, P. A. Forsyth, and G. Labahn. A penalty method for American options with jump diffusion processes. Numer. Math., 97(2):321-352, 2004.

28. G. Duvaut and J.-L. Lions. Les inéquations en mécanique et en physique. Dunod, Paris, 1972. Travaux et Recherches Mathématiques, No. 21.

29. B. Faermann. Efficient and reliable a-posteriori error estimates for boundary element methods. In Analysis, numerics and applications of differential and integral equations (Stuttgart, 1996), volume 379 of Pitman Res. Notes Math. Ser., pages 87-91. Longman, Harlow, 1998.

30. B. Faermann. Efficient and reliable a posteriori error estimates for boundary integral operators of positive and negative order. In ENUMATH 97 (Heidelberg), pages 303-310. World Sci. Publ., River Edge, NJ, 1998.

31. B. Faermann. Local a-posteriori error indicators for the Galerkin discretization of boundary integral equations. Numer. Math., 79(1):43-76, 1998.

32. W. Farkas, N. Reich, and C. Schwab. Anisotropic stable Lévy copula processes - analytical and numerical aspects. MSAS, to appear.

33. F. Fierro and A. Veeser. A posteriori error estimators for regularized total variation of characteristic functions. SIAM J. Numer. Anal., 41(6):2032-2055, 2003.

34. H. Fujita and T. Suzuki. Evolution problems. In Handbook of numerical analysis, Vol. II, Handb. Numer. Anal., II, pages 789-928. North-Holland, Amsterdam, 1991.

35. C. Gräser and R. Kornhuber. Multigrid methods for obstacle problems. J. Comput. Math., 27(1):144, 2009.

36. W. Hackbusch. Integral equations, volume 120 of International Series of Numerical Mathematics. Birkhäuser Verlag, Basel, 1995. Theory and numerical treatment, Translated and revised by the author from the 1989 German original.

37. D. Kinderlehrer and G. Stampacchia. An introduction to variational inequalities and their applications, volume 31 of Classics in Applied Mathematics. Society for Industrial and Applied Mathematics (SIAM), Philadelphia, PA, 2 edition, 2000.

38. R. Kornhuber. Adaptive monotone multigrid methods for nonlinear variational problems. Advances in Numerical Mathematics. B. G. Teubner, Stuttgart, 1997.

39. R. Kress. Linear integral equations, volume 82 of Applied Mathematical Sciences. Springer-Verlag, New York, second edition, 1999.

40. O. Lakkis and C. Makridakis. Elliptic reconstruction and a posteriori error estimates for fully discrete linear parabolic problems. Math. Comp., 75(256):1627-1658 (electronic), 2006.

41. R. Lord, F. Fang, F. Bervoets, and C. W. Oosterlee. A fast and accurate FFT-based method for pricing early-exercise options under Lévy processes. SIAM J. Sci. Comput., 30(4):1678-1705, 2008.

42. A.-M. Matache, P.-A. Nitsche, and C. Schwab. Wavelet Galerkin pricing of American options on Lévy driven assets. Quant. Finance, 5(4):403-424, 2005.

43. A.-M. Matache, T. von Petersdorff, and C. Schwab. Fast deterministic pricing of options on Lévy driven assets. M2AN Math. Model. Numer. Anal., 38(1):37-71, 2004.

44. W. McLean. Strongly elliptic systems and boundary integral equations. Cambridge University Press, Cambridge, 2000. 
45. K.-S. Moon, R. H. Nochetto, T. von Petersdorff, and C.-S. Zhang. A posteriori error analysis for parabolic variational inequalities. Mathematical Modelling and Numerical Analysis, 41(3):485-511, 2007.

46. R. Nochetto, K. Siebert, and A. Veeser. Multiscale, Nonlinear and Adaptive Approximation, chapter Theory of adaptive finite element methods: an introduction. Springer, 2009.

47. R. H. Nochetto, G. Savaré, and C. Verdi. A posteriori error estimates for variable time-step discretizations of nonlinear evolution equations. Comm. Pure Appl. Math., 53(5):525-589, 2000.

48. R. H. Nochetto, A. Schmidt, K. G. Siebert, and A. Veeser. Pointwise a posteriori error estimates for monotone semi-linear equations. Numer. Math., 104(4):515-538, 2006.

49. R. H. Nochetto, K. G. Siebert, and A. Veeser. Pointwise a posteriori error control for elliptic obstacle problems. Numer. Math., 95(1):163-195, 2003.

50. S. Sauter and C. Schwab. Quadrature for hp-Galerkin BEM in $R^{3}$. Numer. Math., 78(2):211-258, 1997.

51. S. A. Sauter and C. Schwab. Randelementmethoden. Teubner, 2004.

52. A. Schmidt and K. G. Siebert. Design of adaptive finite element software, volume 42 of Lecture Notes in Computational Science and Engineering. Springer-Verlag, Berlin, 2005.

53. C. Schwab. Variable order composite quadrature of singular and nearly singular integrals. Computing, 53(2):173-194, 1994.

54. C. Schwab and R. Stevenson. Space-time adaptive wavelet methods for parabolic evolution problems. Math. Comp., (to appear).

55. L. Silvestre. Regularity of the obstacle problem for a fractional power of the Laplace operator. Comm. Pure Appl. Math., 60(1):67-112, 2007.

56. W. Spann. On the boundary element method for the Signorini problem of the Laplacian. Numer. Math., 65(3):337-356, 1993.

57. X.-C. Tai. Rate of convergence for some constraint decomposition methods for nonlinear variational inequalities. Numer. Math., 93(4):755-786, 2003.

58. M. E. Taylor. Pseudodifferential operators, volume 34 of Princeton Mathematical Series. Princeton University Press, Princeton, N.J., 1981.

59. V. Thomée. Galerkin finite element methods for parabolic problems, volume 25 of Springer Series in Computational Mathematics. Springer-Verlag, Berlin, second edition, 2006.

60. A. Veeser. Efficient and reliable a posteriori error estimators for elliptic obstacle problems. SIAM J. Numer. Anal., 39(1):146-167, 2001.

61. R. Verfürth. A review of a posteriori error estimation and adaptive mesh refinement techniques. Wiley and Teubner, 1996.

62. T. von Petersdorff. Randwertprobleme der Elasitizitätstheorie für Polyeder-Singularitäten und Approximation mit Randelementmethoden. Ph.D. thesis, Technische Hochschule Darmstadt, 1989.

63. W. L. Wendland and D. H. Yu. Adaptive boundary element methods for strongly elliptic integral equations. Numer. Math., 53(5):539-558, 1988.

64. W. L. Wendland and D. H. Yu. A posteriori local error estimates of boundary element methods with some pseudo-differential equations on closed curves. J. Comput. Math., 10(3):273-289, 1992.

65. P. Wilmott, J. Dewynee, and S. Howison. Option pricing: mathematical models and computation. Oxford Financial Press, Oxford, UK, 1993. 